\title{
Capacity Bounds for the Gaussian IM-DD Optical Multiple-Access Channel
}

\author{
Anas Chaaban, Member, IEEE, Omer M. S. Al-Ebraheemy, Tareq Y. Al-Naffouri, Member, IEEE, and \\ Mohamed-Slim Alouini, Fellow, IEEE
}

\begin{abstract}
Optical wireless communications (OWC) is a promising technology for closing the mismatch between the growing number of connected devices and the limited wireless network capabilities. Similar to downlink, uplink can also benefit from OWC for establishing connectivity between such devices and an optical access point. In this context, the incoherent intensitymodulation and direct-detection (IM-DD) scheme is desirable in practice. Hence, it is important to understand the fundamental limits of communication rates over an OWC uplink employing IM-DD, i.e., the channel capacity. This uplink, modeled as a Gaussian multiple-access channel (MAC) for indoors OWC, is studied in this paper, under the IM-DD constraints which form the main difference with the standard Gaussian MAC commonly studied in the radio-frequency context. Capacity region outer and inner bounds for this channel are derived. The bounds are fairly close at high signal-to-noise ratio (SNR), where a truncatedGaussian input distribution achieves the capacity region within a constant gap. Furthermore, the bounds coincide at low SNR showing the optimality of on-off keying combined with successive cancellation decoding in this regime. At moderate SNR, an optimized uniformly-spaced discrete input distribution achieves fairly good performance.
\end{abstract}

Index Terms-Intensity-modulation, multiple-access, capacity bounds, successive cancellation, capacity approximation.

\section{INTRODUCTION}

Radio-frequency (RF) communication systems are becoming unable to cope with the rapidly increasing demand for high data-rates. This motivated researchers to explore other opportunities, such as optical wireless communications (OWC) [2]-[4]. The abundance of the optical spectrum makes it a perfect candidate for achieving this goal, both outdoors and indoors. It can be used outdoors to establish links between base-stations or buildings, over single or multiple hops using lasers [5]-[8]. On the other hand, it can be used indoors to establish LiFi (light fidelity) optical attocells using lightemitting diodes (LED) [9], [10]. The inherent properties of OWC allows for dense frequency and spatial reuse, leading to

A. Chaaban, T. Y. Al-Naffouri, and M.-S. Alouini are with the Division of Computer, Electrical, and Mathematical Sciences and Engineering (CEMSE) at King Abdullah University of Science and Technology (KAUST), Thuwal, Saudi Arabia. Email: \{anas.chaaban,tareq.alnaffouri,slim.alouini\}@kaust.edu.sa. Their work was supported in part by the Qatar National Research Fund (a member of Qatar Foundation) under Grant NPRP 9-077-2-036. The statements made herein are solely the responsibility of the authors.

O. M. S. Al-Ebraheemy is with the Department of Electrical Engineering, King Fahd University of Petroleum and Minerals, Dhahran 31261, Saudi Arabia. Email: omermahmoud@kfupm.edu.sa.

Part of this work was presented at ISCAS 2016 [1]. high data-rates per unit area without interfering with available RF systems [9].

\section{A. Multi-user Optical Wireless Communications}

Indoors OWC can be used for serving multiple nodes (users, devices, etc.) in downlink (DL) or uplink (UL). DL can be implemented using visible-light communications (VLC) through LED light fixtures, which efficiently combines lighting and communication functionalities. Another alternative is to use infrared LEDs. The latter is more suitable for UL between multiple nodes and a common receiver [9], [11], since it is invisible to the human eye. In both cases, very high data-rates (hundreds of megabits/second [12]) can be achieved due to the high switching speeds of LEDs.

Multi-user OWC has received lots of research attention recently [13]-[23]. For instance, [13]-[15] proposed power allocation strategies to enhance the throughput of VLC DL. Ma et al. [16] proposed coordination techniques for improving the performance of VLC attocells. The performance of different multiple access techniques for VLC DL was studied in [17][21], and capacity region analysis was considered in [22].

In addition to DL, UL is of fundamental importance when it comes to a complete LiFi system [9]. While UL can be established using RF, offloading this traffic to the optical band is desirable due to the higher data-rates of OWC and its locality. Consider for instance a large number of transmitters spread over multiple rooms within the coverage of a single RF access point. All transmitters will compete for the RF uplink channels, which limits the number of served transmitters or their data-rates. Suppose now that each room has its own optical attocell access point. The high data-rate of optical UL allows serving many transmitters without disturbing the RF system. Thus, one can offload traffic of the RF UL to the optical UL, consequently achieving better performance in both systems. The overall system can support more transmitters and higher rates than a system with an RF UL only. This is particularly important in light of the increased interest in the internet-of-things [24] and the envisioned increase of the number of connected devices in the future. This is also important for supporting future applications which require high uplink data-rates such as virtual and holographic telepresence. Additionally, OWC UL is useful for establishing optical local-area networks [11], which in addition to high rates and dense spatial reuse, enjoy enhanced security, and low 
electromagnetic interference which is desirable in applications in hospitals and airplanes for example.

\section{B. Objective}

To understand the capabilities of OWC UL, we study its capacity in this paper. We consider an OWC UL where transmitters communicate with a receiver using intensity-modulation and direct-detection (IM-DD). This scheme is favorable in practice due to its simplicity. The capacity of this multipleaccess channel (MAC) is interesting from both theoretical and practical perspectives. From a theoretical perspective, this channel is different from RF MAC due to the use of intensity modulation, and hence its capacity is different. From a practical perspective, capacity is the fundamental limit on the achievable data-rates over a given channel, and hence, is an important design benchmark. Furthermore, studying capacity highlights opportunities that can be exploited in practice such as the optimal transmission schemes.

To this end, we use an additive channel with independent Gaussian noise to model an indoors IM-DD optical MAC (OMAC) similar to [11]. We consider both average and peak intensity constraints at the transmitters. Since multiple transmitters send to the same receiver, their signals naturally interference, which is the main difference with [25], [26]. Indeed, this interference can be avoided by using orthogonal codes, similar to [23], [27], [28] for instance. However, orthogonal transmission is generally not optimal from a capacity point of view. It is better to use nonorthogonal multiple access and use other techniques to handle interference such as successivecancellation decoding (SCD) [29].

The capacity region of the discrete-memoryless MAC has been characterized in [30], [31]. Applied to the OMAC, this characterization is expressed as the convex-hull of the union of rate regions over the set of input distributions that satisfy the nonnegativity, average, and peak intensity constraints of IM-DD. While this union simplifies in the RF Gaussian MAC (with a power constraint) where the optimal input distribution is known, this is not the case in IM-DD. In this case, the optimal input distribution is unknown (even for the IMDD point-to-point (P2P) channel [25], [26]) and calculating this union is prohibitive. Nevertheless, to obtain computable descriptions of the capacity region of the OMAC, one can resort to capacity bounds.

\section{Summary of Contributions}

We derive capacity outer bounds for the OMAC expressed in terms of capacity upper bounds for the IM-DD P2P channel in [25], [32]. The bounds have simple representations at high and low signal-to-noise ratios (SNR). Then, we derive an inner bound based on truncated-Gaussian (TG) input distributions [32] in addition to SCD and time-sharing. To express this bound, we derive the achievable rate in a channel with a TG input and a mixture of Gaussian noise and TG interference. We also provide an inner bound based on a uniformly-spaced discrete input distribution [33], which is optimized in order to enlarge the achievable rate region. Additionally, we prove that it suffices to consider an average intensity constraint smaller

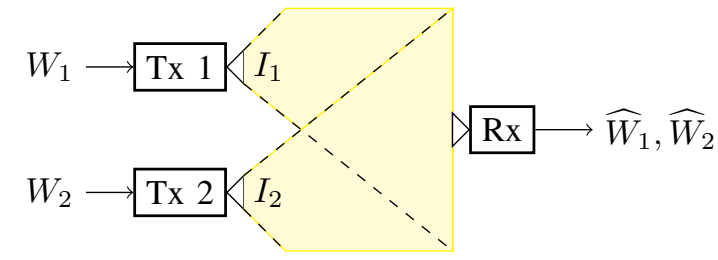

Fig. 1: Optical multiple-access channel with two transmitters. Tx $i$ sends message $W_{i}$ by modulating light intensity $I_{i}$, and Rx processes the received signal to decode $\widehat{W}_{i}$.

than half the peak intensity constraint in the OMAC, similar to the IM-DD P2P channel [25] and the IM-DD broadcast channel [22].

We then compare the bounds at high, low, and moderate SNR. At high SNR, the TG distribution achieves capacity within a small gap. We characterize this gap and obtain a simple capacity approximation at high SNR. We also approximate the symmetric capacity within a gap that vanishes as the number of transmitters grows. At low SNR, the a discrete input achieves the best performance, converging to capacity as SNR decreases. In this case, the capacity region is rectangular characterized by simple expressions, and achievable by onoff keying combined with SCD. At moderate SNR, a discrete input with a small alphabet (binary, ternary, or quaternary) suffices to achieve good performance. We also compare the achievable rates with orthogonal time-division multiple access (TDMA) transmission, to demonstrate the inferiority of TDMA in the OMAC.

\section{Paper Organization and Notations}

The rest of the paper is organized as follows. Sec. II provides the notation used in the paper and the considered channel model. Capacity region outer bounds for the OMAC with two transmitters are given in Sec. III and capacity region inner bounds in Sec. IV. The bounds are compared and capacity statements are obtained in Sec. V. The results are extended to the $N$-transmitter OMAC in Sec. VI and the paper is concluded in Sec. VII.

Throughout the paper, $g_{\mu, \nu}(x)$ and $G_{\mu, \nu}(x)$ are used to represent the probability density function (PDF) and cumulative density function (CDF) of a Gaussian random variable with mean $\mu$ and variance $\nu^{2}$, respectively. Normal-face fonts represent scalars and bold-face represent vectors. Furthermore, $\mathrm{CH}(\cdot)$ is use to denote the convex hull of a set, and $[x]^{+}$to denote $\max \{0, x\}$.

\section{The IM-DD Optical Multiple-Access Channel}

\section{A. Channel Model}

In the IM-DD optical MAC (Fig. 1), transmitter $i \in \mathcal{N}=$ $\{1, \ldots, N\}$ uses light intensity to transmit data to a common optical receiver. Using digital transmission, the system can be represented by a discrete-time channel with transmitter intensity denoted $I_{i}(t) \geq 0$ and received signal denoted $S(t)$ at time instant $t \in \mathbb{N}$. The transmitter has a peak intensity constraint $I_{i}(t) \leq I_{i, \max }$ and an average intensity constraint 
$\mathbb{E}\left[I_{i}(t)\right] \leq \bar{I}_{i} \forall t$, due to practical and safety reasons. The received signal can be written as [11]

$$
S(t)=r \sum_{i=1}^{N} h_{i}(t) I_{i}(t)+N(t),
$$

where $r$ is the receiver sensitivity, $h_{i}(t)$ is the channel coefficient, and $N(t)$ is noise. The photonic devices are assumed to be linear in the regime of operation.

We focus on indoors scenarios, and we assume the existence of a significantly dominant path between each transmitter and the receiver, i.e., negligible multipath. This can be the case if the system is directed (with directional transmitters ${ }^{1}$ ), or nondirected with either an nonshadowed line-of-sight (LOS) or with diffuse operation [11]. Note that indoors OWC is not susceptible to scintillation as outdoors scenarios. Moreover, mobility (if any) leads to very slow channel variations relative to the symbol rate for most indoor applications [34], and hence can be neglected. Thus, the channel $h_{i}(t)$ can be assumed to be constant over the duration of interest (transmission duration), i.e., $h_{i}(t)=h_{i} \forall t$.

Noise $N(t)$ has zero mean and variance $\sigma^{2}$, and combines thermal noise, relative intensity noise, and ambient light shot noise. This can be well modeled as Gaussian and independent of the transmit signals [2], [11], [25], [35], [36]. This independence is reasonable in scenarios with strong ambient light and/or thermal noise [36], [37].

This input-output relation for a given $t$ can be expressed as

$$
Y=\sum_{i=1}^{N} X_{i}+Z,
$$

by defining $r h_{i} I_{i}(t), S(t)$, and $N(t)$ as random variables $X_{i}$, $Y$ and $Z$. We focus on this model for it has the same capacity as the model in (1). We can interpret $X_{i}$ as the received optical intensity from transmitter $i$ at a given transmission. The constraints on $I_{i}$ lead to $X_{i} \geq 0, X_{i} \leq \mathcal{A}_{i}$, and $\mathbb{E}\left[X_{i}\right] \leq \mathcal{E}_{i}{ }^{2}{ }^{2}$ where $\mathcal{A}_{i}=r h_{i} I_{i, \max }$ and $\mathcal{E}_{i}=r h_{i} \bar{I}_{i}$. We denote the ratio $\frac{\mathcal{E}_{i}}{\mathcal{A}_{i}}$ by $\alpha_{i}$ with $\alpha_{i} \in\left[0, \frac{1}{2}\right]^{3}$. We assume without loss of generality that $\mathcal{A}_{1} \geq \mathcal{A}_{2} \geq \cdots \geq \mathcal{A}_{N}$.

We say that the channel has high SNR if $\mathcal{E}_{i} \gg \sigma$ and consequently $\mathcal{A}_{i} \gg \sigma$, for all $i$. Conversely, we say that the channel has low SNR if $\mathcal{A}_{i} \ll \sigma$ and consequently $\mathcal{E}_{i} \ll \sigma$, for all $i$.

\section{B. Coding and Capacity}

A general coding scheme for the OMAC can be described as follows. Transmitter $i$ wants to send a message $W_{i}$ chosen uniformly from $\mathcal{W}_{i}=\left\{1, \ldots,\left|\mathcal{W}_{i}\right|\right\}$ to the receiver. This message is encoded to a codeword of length $n \in \mathbb{N}$ denoted $\boldsymbol{X}_{i} \in \mathbb{R}_{+}^{n}$, and then transmitted, one symbol at a time. The codewords at transmitter $i$ constitute a codebook that must satisfy the average and peak constraints $\mathcal{E}_{i}$ and $\mathcal{A}_{i}$. The receiver collects

\footnotetext{
${ }^{1}$ A slightly divergent beam can be used to reduce the effect of misalignment.

${ }^{2}$ These constraints are the main differences between with the RF MAC.

${ }^{3}$ Generally, the average constraint is redundant if $\alpha_{i} \geq 1$. However, as we show in Appendix A, it suffices to consider $\alpha_{i} \leq \frac{1}{2}$ to capture the whole range of $\alpha_{i} \in[0,1]$.
}

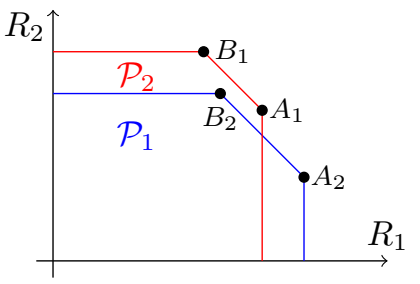

Fig. 2: An example of MAC achievable rate regions (pentagons $\mathcal{P}_{1}$ and $\mathcal{P}_{2}$ ) for two different input distributions.

the received symbols over $n$ transmission in $\boldsymbol{Y} \in \mathbb{R}^{n}$, and then uses a decoder to decode $\widehat{W}_{i} \in \mathcal{W}_{i}$, for all $i \in \mathcal{N}$, from $\boldsymbol{Y}$. The transmission rate from transmitter $i$ to the receiver is then defined as $R_{i}=\frac{\log \left(\left|\mathcal{W}_{i}\right|\right)}{n}$ in nats/transmission. ${ }^{4}$

We are interested in the set of achievable rate tuples $\left(R_{1}, \ldots, R_{N}\right)$ so that the error probability $\operatorname{Pr}\left\{W_{i} \neq \widehat{W}_{i}, i \in\right.$ $\mathcal{N}\}$ can be made arbitrarily small by increasing the code length $n$. The set of all achievable rate tuples is the capacity region denoted $\mathcal{C}$. The symmetric capacity is defined as $C_{\mathrm{s}}=\max _{(R, \ldots, R) \in \mathcal{C}} R$.

The capacity region of a discrete-memoryless MAC is known from the works of Ahlswede [30] and Liao [31]. For $N=2$, the channel is defined by a conditional probability $p\left(y \mid x_{1}, x_{2}\right)$ with $y \in \mathcal{Y}$ and $x_{i} \in \mathcal{X}_{i}$, with $\mathcal{Y}$ and $\mathcal{X}_{i}$ being the output and input alphabets, respectively. Its capacity region $\mathcal{C}$ is given as the closure of the convex-hull of $\left(R_{1}, R_{2}\right) \in \mathbb{R}_{+}^{2}$ satisfying [38]

$$
\begin{aligned}
R_{1} & \leq I\left(X_{1} ; Y \mid X_{2}\right) \\
R_{2} & \leq I\left(X_{2} ; Y \mid X_{1}\right) \\
R_{1}+R_{2} & \leq I\left(X_{1}, X_{2} ; Y\right)
\end{aligned}
$$

for some input distribution $p\left(x_{1}, x_{2}\right)=p_{1}\left(x_{1}\right) p_{2}\left(x_{2}\right)$ over $\mathcal{X}_{1} \times \mathcal{X}_{2}$ (see Fig. 2). This capacity region is achievable by jointly decoding $\left(W_{1}, W_{2}\right)$ at the receiver, or by successive decoding combined with time-sharing. In successive decoding, the receiver decodes $W_{1}\left(W_{2}\right)$ first, then it decodes $W_{2}\left(W_{1}\right)$ with knowledge of $\boldsymbol{X}_{1}\left(\boldsymbol{X}_{2}\right)$, thus achieving the point $B_{1}$ $\left(A_{1}\right)$ in Fig. 2 for instance. By time sharing between the two decoding orders (points $B_{1}$ and $\left.A_{1}\right), \mathcal{C}$ can be achieved.

This statement can be applied to the OMAC with continuous alphabets using the discretization procedure in [38, Sec. 3.4], as stated in [38, Sec. 4.6.1]. In this case, we replace $\mathcal{X}_{1}$ and $\mathcal{X}_{2}$ by $\mathbb{R}_{+}, \mathcal{Y}$ by $\mathbb{R}$, we define $p_{i}\left(x_{i}\right)$ as a probability mass function that satisfies the intensity constraints, and write the mutual information $I\left(X_{1} ; Y \mid X_{2}\right)$ as $h\left(Y \mid X_{2}\right)-h\left(Y \mid X_{1}, X_{2}\right)$, i.e., in terms of differential entropies instead of entropies.

Unfortunately, computing this region is prohibitively complex, since the optimal $p\left(x_{1}, x_{2}\right)$ is unknown. Thus, instead of evaluating this region, we seek computable bounds on it from which we can draw conclusions and comment of the optimality of some schemes. We start with the 2-transmitter OMAC, and we extend the results and insights to the $N$-transmitter case in Sec. VI. The following section presents outer bounds on the capacity region for $N=2$.

\footnotetext{
${ }^{4}$ All logarithms in the paper are natural logarithms.
} 


\section{CAPACITy REgion OUter Bounds}

Capacity region outer bounds provide necessary conditions for the achievability of a rate pair $\left(R_{1}, R_{2}\right)$. Such bounds are derived in this section. But before we proceed, we introduce upper bounds on the capacity of the IM-DD point-to-point channel which is instrumental for this purpose.

\section{A. P2P Capacity Upper Bounds}

Consider a P2P channel where a transmitter wants to send a message to a receiver using IM-DD. The channel has input $X$, output $Y$ and noise $Z$, where

$$
\begin{aligned}
& Y=X+Z, \quad Z \sim g_{0, \sigma}(z), \\
& X \in[0, \mathcal{A}], \quad \mathbb{E}[X] \leq \mathcal{E}=\alpha \mathcal{A}, \quad \alpha \leq \frac{1}{2} .
\end{aligned}
$$

The capacity of this channel has been studied in [25], [26], [32], [33]. The following bounds on the capacity of this channel will be useful in the sequel.

Lemma 1: The capacity of the IM-DD P2P channel defined in (4) is upper bounded by the following quantities

$$
\begin{aligned}
& \bar{C}_{\alpha}^{[1]}(\mathcal{A}, \sigma)=\inf _{\beta, \delta>0} B_{1}(\beta, \delta), \\
& \bar{C}_{\alpha}^{[2]}(\mathcal{A}, \sigma)=\sup _{\delta \in[0,1]} B_{2}(\delta), \\
& \bar{C}_{\alpha}^{[3]}(\mathcal{A}, \sigma)=\frac{1}{2} \log \left(1+\frac{\alpha(1-\alpha) \mathcal{A}^{2}}{\sigma^{2}}\right),
\end{aligned}
$$

where $B_{1}(\beta, \delta)$ is given in (9) at the top of next page wherein $Q(x)=\frac{1}{\sqrt{2 \pi}} \int_{x}^{\infty} e^{-\frac{t^{2}}{2}} d t$, and where

$$
B_{2}(\delta)=\delta \log \left[\frac{\mathcal{A}}{\sqrt{2 \pi e} \sigma}\right]-\log \left[\delta^{\delta}(1-\delta)^{\frac{3(1-\delta)}{2}}\right] .
$$

The first upper bound was given in [25] and is asymptotically tight at high SNR when the peak constraint is inactive. Conversely, the second bound which was given in [32] is asymptotically tight at high SNR when the average constraint is inactive. The last one was given in [25] and is asymptotically tight at low SNR. In general, none of those bounds dominates the others over the whole range of $\alpha, \mathcal{E}$, and $\mathcal{A}$.

The asymptotic behavior of the bounds $\bar{C}_{\alpha}^{[1]}(\mathcal{A}, \sigma)$ and $\bar{C}_{\alpha}^{[2]}(\mathcal{A}, \sigma)$ will also be needed in what follows. These bounds approach $\frac{1}{2} \log \left(1+\frac{e \alpha^{2} \mathcal{A}^{2}}{2 \pi \sigma^{2}}\right)$ and $\frac{1}{2} \log \left(1+\frac{\mathcal{A}^{2}}{2 \pi e \sigma^{2}}\right)$, respectively, as $\mathcal{A}$ grows [25], [32]. We combine the two asymptotes into the following expression

$$
\begin{aligned}
\bar{C}_{\alpha}^{[\mathrm{h}]}(\mathcal{A}, \sigma) & =\frac{1}{2} \log \left(1+\frac{c(\alpha) \mathcal{A}^{2}}{\sigma^{2}}\right), \\
c(\alpha) & =\min \left\{\frac{1}{2 \pi e}, \frac{e \alpha^{2}}{2 \pi}\right\} .
\end{aligned}
$$

This expression will be used to describe the asymptotic behavior of the capacity region of the OMAC at high SNR.

Now, we are ready to present the OMAC capacity region outer bounds.

\section{B. OMAC Capacity Region Outer Bounds}

It is convenient to derive capacity region bounds which have a simple geometry, which is simple to analyze. Bounds which have the form of a convex polytope are a example. The following theorem provides a computable capacity outer bound based on Lemma 1, which has such a structure.

Theorem 1: The capacity region $\mathcal{C}$ of the OMAC with 2 transmitters satisfies $\mathcal{C} \subseteq \overline{\mathcal{C}}^{[j]}, j \in\{1,2,3\}$, defined as the set of $\left(R_{1}, R_{2}\right) \in \mathbb{R}_{+}^{2}$ satisfying

$$
\begin{aligned}
R_{i} & \leq \bar{C}_{\alpha_{i}}^{[j]}\left(\mathcal{A}_{i}, \sigma\right), i \in\{1,2\} \\
R_{1}+R_{2} & \leq \bar{C}_{\alpha_{12}}^{[j]}\left(\mathcal{A}_{1}+\mathcal{A}_{2}, \sigma\right),
\end{aligned}
$$

where $\alpha_{12}=\frac{\mathcal{E}_{1}+\mathcal{E}_{2}}{\mathcal{A}_{1}+\mathcal{A}_{2}}$.

Proof: For $R_{1}$ to be achievable, it has to satisfy $R_{1}<$ $I\left(X_{1} ; Y \mid X_{2}\right)(3)$. But

$$
\begin{aligned}
I\left(X_{1} ; Y \mid X_{2}\right) & =h\left(Y \mid X_{2}\right)-h\left(Y \mid X_{1}, X_{2}\right) \\
& =h\left(X_{1}+Z\right)-h(Z) \\
& \leq \max _{p_{1}\left(x_{1}\right)} I\left(X_{1} ; X_{1}+Z\right)
\end{aligned}
$$

where the maximization is over distributions of $X_{1} \in\left[0, \mathcal{A}_{1}\right]$ that satisfy $\mathbb{E}\left[X_{1}\right] \leq \mathcal{E}_{1}$. But this upper bound is the capacity of an IM-DD P2P channel with input $X_{1}$ and output $X_{1}+Z$. By Lemma 1 , this is upper bounded by $\bar{C}_{\alpha_{1}}^{[j]}\left(\mathcal{A}_{1}, \sigma\right)$ leading to the first bound. The second bound can be obtained similarly. On the other hand, a rate pair $\left(R_{1}, R_{2}\right)$ is achievable if $R_{1}+R_{2}<$ $I\left(X_{1}, X_{2} ; Y\right)$. But

$$
\begin{aligned}
I\left(X_{1}, X_{2} ; Y\right) & =h(Y)-h\left(Y \mid X_{1}, X_{2}\right) \\
& =h(Y)-h(Z) \\
& =h(Y)-h\left(Y \mid X_{1}+X_{2}\right) \\
& =I(X ; Y),
\end{aligned}
$$

where $X=X_{1}+X_{2}$, which follows due to the independence of $Z$ and $X_{i}$. Note that $I(X ; Y) \leq \max _{p(x)} I(X ; Y)$ where the maximization is subject to $X \in\left[0, \mathcal{A}_{1}+\mathcal{A}_{2}\right]$ satisfying $\mathbb{E}[X] \leq \alpha_{12}\left(\mathcal{A}_{1}+\mathcal{A}_{2}\right)$. This upper bound is the capacity of a Gaussian IM-DD P2P channel with a peak constraint $\mathcal{A}_{1}+\mathcal{A}_{2}$ and an average constraint $\alpha_{12}\left(\mathcal{A}_{1}+\mathcal{A}_{2}\right)$, which is upper bounded by $\bar{C}_{\alpha_{12}}^{[j]}\left(\mathcal{A}_{1}+\mathcal{A}_{2}, \sigma\right)$ by Lemma 1 . This concludes the proof of the theorem.

Note that the main new ingredient in the derivation of this bound is the representation of the sum-rate bound on $R_{1}+R_{2}$ in the form of an IM-DD P2P channel upper bound. This is done by defining $X_{1}+X_{2}$ as a new random variable with nonnegativity, average, and peak intensity constraints.

Due to (10), the outer bounds $\mathcal{C} \subseteq \overline{\mathcal{C}}^{[1]}$ and $\mathcal{C} \subseteq \overline{\mathcal{C}}^{[2]}$ can be combined to a single outer bound which converges to a simple region as SNR increases. This is provided in the following corollary.

Corollary 1: The capacity region of the 2-transmitter OMAC is outer bounded by $\overline{\mathcal{C}}^{[12]}=\overline{\mathcal{C}}^{[1]} \cap \overline{\mathcal{C}}^{[2]}$. Furthermore, the region 


$$
B_{1}(\beta, \delta)=\log \left[\frac{\beta e^{-\frac{\delta^{2}}{2 \sigma^{2}}}}{\sqrt{2 \pi e} \sigma}+\frac{Q\left(\frac{\delta}{\sigma}\right)}{\sqrt{e}}\right]+\frac{Q\left(\frac{\delta}{\sigma}\right)}{2}+\frac{\left(\frac{\delta}{2 \sigma}+\frac{\sigma}{\beta}\right) e^{-\frac{\delta^{2}}{2 \sigma^{2}}}}{\sqrt{2 \pi}}+\frac{\delta^{2}\left(1-Q\left(\frac{\delta+\mathcal{E}}{\sigma}\right)\right)}{2 \sigma^{2}}+\frac{\delta+\mathcal{E}}{\beta}
$$

$\overline{\mathcal{C}}^{[12]}$ converges $^{5}$ as SNR increases to $\overline{\mathcal{C}}^{[\mathrm{h}]}$ defined as the set of $\left(R_{1}, R_{2}\right) \in \mathbb{R}_{+}^{2}$ satisfying

$$
\begin{aligned}
R_{i} & \leq \frac{1}{2} \log \left(1+\frac{c\left(\alpha_{i}\right) \mathcal{A}_{i}^{2}}{\sigma^{2}}\right), i \in\{1,2\}, \\
R_{1}+R_{2} & \leq \frac{1}{2} \log \left(1+\frac{c\left(\alpha_{12}\right)\left(\mathcal{A}_{1}+\mathcal{A}_{2}\right)^{2}}{\sigma^{2}}\right),
\end{aligned}
$$

where $c(\alpha)$ is as defined in (11).

Proof: Since both $\overline{\mathcal{C}}^{[1]}$ and $\overline{\mathcal{C}}^{[2]}$ are outer bounds, then an achievable $\left(R_{1}, R_{2}\right)$ must lie in both regions, and hence, their intersection is also an outer bound. Now this intersection is a convex polytope defined by the axes and three bounding lines: $R_{i}=\min \left\{\overline{\mathcal{C}}_{\alpha_{i}}^{[1]}\left(\mathcal{A}_{i}, \sigma\right), \overline{\mathcal{C}}_{\alpha_{i}}^{[2]}\left(\mathcal{A}_{i}, \sigma\right)\right\}$ for $i \in\{1,2\}$, and $R_{1}+R_{2}=\min \left\{\overline{\mathcal{C}}_{\alpha_{12}}^{[1]}\left(\mathcal{A}_{1}+\mathcal{A}_{2}, \sigma\right), \overline{\mathcal{C}}_{\alpha_{12}}^{[2]}\left(\mathcal{A}_{1}+\mathcal{A}_{2}, \sigma\right)\right\}$. Consider the boundary on $R_{i}$. By (10), this boundary converges to the right-hand side of (19) as SNR increases. Similarly, the boundary on $R_{1}+R_{2}$ converge to the right-hand sides of (20) as SNR increases. Thus, the region $\overline{\mathcal{C}}^{[12]}$ converges to $\overline{\mathcal{C}}^{[\mathrm{h}]}$ (also a convex polytope) as SNR increases, which concludes the proof.

This corollary combines $\overline{\mathcal{C}}^{[1]}$ and $\overline{\mathcal{C}}^{[2]}$ into a simple expression at high SNR. The remaining bound $\overline{\mathcal{C}}^{[3]}$ already has a simple expression, and needs no further simplification. We now turn our attention towards capacity inner bounds for comparison with the established outer bounds.

\section{Achievable Rate Regions}

Inner bounds on the capacity region provide sufficient conditions for the achievability of a rate pair $\left(R_{1}, R_{2}\right)$, i.e., every rate pair inside an inner bound is achievable. An inner bound for the capacity region $\mathcal{C}$ can be obtained by calculating the union of (3) over a particular subset of permissible independent input distributions. The best distribution is not known. However, there are some known distributions which perform good enough in some SNR ranges in the P2P channel. We use these distributions to establish capacity inner bounds next. Next, we establish capacity region inner bounds based on two input distributions: A truncated-Gaussian input distribution, and a uniformly-spaced discrete distribution.

\section{A. Truncated-Gaussian Input Distribution}

Using a truncated-Gaussian (TG) input distribution is motivated by its near-optimality at high SNR in the IM-DD P2P channel [32]. A TG distribution has a PDF

$$
\tilde{g}(x)=\eta g_{\mu, \nu}(x) \text { for } x \in[0, \mathcal{A}],
$$

\footnotetext{
${ }^{5}$ We say that two convex polytope regions $\mathcal{R}_{1}$ and $\mathcal{R}_{2}$ converge as SNR grows if the distance between their boundaries vanishes as SNR grows.
}

and $\tilde{g}(x)=0$ elsewhere, where $\eta=\left(G_{\mu, \nu}(\mathcal{A})-G_{\mu, \nu}(0)\right)^{-1}$ is a scaling factor that guarantees that $\int \tilde{g}(x) d x=1$. It is completely characterized by $\mu$ and $\nu$ (the mean and standard deviation of the entailed Gaussian distribution), and by its support $[0, \mathcal{A}]$. Thus, we call it a $(\mu, \nu, \mathcal{A})$-TG distribution. The mean and variance of this distribution are respectively

$$
\begin{aligned}
\tilde{\mu} & =\nu^{2}(\tilde{g}(0)-\tilde{g}(\mathcal{A}))+\mu, \\
\tilde{\nu}^{2} & =\nu^{2}(1-\mathcal{A} \tilde{g}(\mathcal{A})-\tilde{\mu}(\tilde{g}(0)-\tilde{g}(\mathcal{A}))) .
\end{aligned}
$$

We denote the mean, variance, PDF, and scaling factor of a $\left(\mu_{i}, \nu_{i}, \mathcal{A}_{i}\right)$-TG distribution as $\tilde{\mu}_{i}, \tilde{\nu}_{i}^{2}, \tilde{g}_{i}(x)$, and $\eta_{i}$, respectively.

To evaluate the achievable rate region in the OMAC when the transmitters use a TG input distribution, we use the successive-decoding rates. Recall that the region in (3) can be achieved using successive-decoding [39]. This can be seen by writing $I\left(X_{1}, X_{2} ; Y\right)$ as $I\left(X_{1} ; Y\right)+I\left(X_{2} ; Y \mid X_{1}\right)$ or $I\left(X_{2} ; Y\right)+I\left(X_{1} ; Y \mid X_{2}\right)$ using the chain rule. The two summands in each case are the achievable rates using successivedecoding. For instance, if the receiver decodes $W_{1}$ first followed by $W_{2}$, then a rate pair $\left(R_{1}, R_{2}\right)$ is achievable if

$$
R_{1} \leq I\left(X_{1} ; Y\right) \text { and } R_{2} \leq I\left(X_{2} ; Y \mid X_{1}\right) .
$$

Evaluating $I\left(X_{1} ; Y\right)$ and $I\left(X_{2} ; Y \mid X_{1}\right)$ leads to an evaluation of the achievable rate region. To this end, we shall need the following lemmas.

Lemma 2: In the IM-DD P2P channel defined in (4), a $(\mu, \nu, \mathcal{A})$-TG input distribution satisfying $\tilde{\mu} \leq \mathcal{E}$ achieves a rate $R=I(X ; Y) \geq \frac{1}{2} \log \left(\frac{\nu^{2}}{\tilde{\nu}^{2}}+\frac{\nu^{2}}{\sigma^{2}}\right)-\phi$, where $\phi=$ $\log (\eta)+\frac{1}{2}((\mathcal{A}-\mu) \tilde{g}(\mathcal{A})+\mu \tilde{g}(0))$.

This rate has been derived in [22, Theorem 2]. This is useful for evaluating $I\left(X_{2} ; Y \mid X_{1}\right)$ when the inputs at TG-distributed. For evaluating $I\left(X_{1} ; Y\right)$, we need the following.

Lemma 3: In a channel $Y=X+\tilde{Z}$ where $\tilde{Z}=W+Z, Z \sim$ $g_{0, \sigma}(z)$, and $W$ is $\left(\mu_{w}, \nu_{w}, \mathcal{A}_{w}\right)$-TG distributed, a $(\mu, \nu, \mathcal{A})$ TG distributed $X$ with $\tilde{\mu} \leq \mathcal{E}$ achieves a rate $R=I(X ; Y) \geq$ $\frac{1}{2} \log \left(\frac{\nu^{2}}{\tilde{\nu}^{2}}+\frac{\nu^{2}}{\tilde{\nu}_{w}^{2}+\sigma^{2}}\right)-\phi$, where $\tilde{\mu}, \tilde{\nu}^{2}$, and $\tilde{\nu}_{w}^{2}$ are the mean of $X(22)$, its variance, and the variance of $W$ (23), respectively, and $\phi$ is as defined in Lemma 2.

Proof: We first write the achievable rate as $R=$ $I(X ; Y)=h(X)-h(X \mid Y)$. Since $X$ is $(\mu, \nu, \mathcal{A})$-TG distributed, its entropy is given by $h(X)=\frac{1}{2} \log \left(2 \pi e \nu^{2}\right)-\phi$. Now consider $h(X \mid Y)$. Since $X$ and $W$ are TG-distributed, their variances are given by (23) as

$$
\begin{aligned}
\tilde{\nu}^{2} & =\nu^{2}(1-\mathcal{A} \tilde{g}(\mathcal{A})-\tilde{\mu}(\tilde{g}(0)-\tilde{g}(\mathcal{A}))) \\
\tilde{\nu}_{w}^{2} & =\nu_{w}^{2}\left(1-\mathcal{A}_{w} \tilde{g}\left(\mathcal{A}_{w}\right)-\tilde{\mu}_{w}\left(\tilde{g}(0)-\tilde{g}\left(\mathcal{A}_{w}\right)\right)\right),
\end{aligned}
$$

respectively, where $\tilde{\mu}=\nu^{2}(\tilde{g}(0)-\tilde{g}(\mathcal{A}))+\mu$ and $\tilde{\mu}_{w}=$ $\nu_{w}^{2}\left(\tilde{g}(0)-\tilde{g}\left(\mathcal{A}_{w}\right)\right)+\mu_{w}$. The covariance matrix of $(X, Y)$ is given by $S=\left[\begin{array}{cc}\tilde{\nu}^{2} & \tilde{\nu}^{2} \\ \tilde{\nu}^{2} & \tilde{\nu}^{2}+\tilde{\nu}_{w}^{2}+\sigma^{2}\end{array}\right]$. Under this covariance 
constraint, the conditional differential entropy $h(X \mid Y)$ is maximized by jointly Gaussian $(X, Y)$ [40]. This leads to

$$
h(X \mid Y) \leq \frac{1}{2} \log \left(\frac{2 \pi e|\boldsymbol{S}|}{\operatorname{var}(Y)}\right)=\frac{1}{2} \log \left(2 \pi e \frac{\tilde{\nu}^{2}\left(\tilde{\nu}_{w}^{2}+\sigma^{2}\right)}{\tilde{\nu}^{2}+\tilde{\nu}_{w}^{2}+\sigma^{2}}\right),
$$

where $\operatorname{var}(Y)=\tilde{\nu}^{2}+\tilde{\nu}_{w}^{2}+\sigma^{2}$ is the variance of $Y$. Therefore, we have that

$$
\begin{aligned}
R & \geq \frac{1}{2} \log \left(2 \pi e \nu^{2}\right)-\phi+\frac{1}{2} \log \left(2 \pi e \frac{\tilde{\nu}^{2}+\tilde{\nu}_{w}^{2}+\sigma^{2}}{\tilde{\nu}^{2}\left(\tilde{\nu}_{w}^{2}+\sigma^{2}\right)}\right) \\
& =\frac{1}{2} \log \left(\frac{\nu^{2}}{\tilde{\nu}^{2}}+\frac{\nu^{2}}{\tilde{\nu}_{w}^{2}+\sigma^{2}}\right)-\phi .
\end{aligned}
$$

This concludes the proof.

With these two lemmas, we are ready to express an achievable rate region.

1) Achievable Rate Region: The following theorem provides an achievable rate region based on TG-distributed inputs, which establishes a capacity region inner bound.

Theorem 2: The rate region $\mathcal{R}_{\mathrm{T}}=\mathrm{CH}\left(\bigcup_{\boldsymbol{\mu}, \boldsymbol{\nu}} \mathcal{R}_{\mathrm{t}}(\boldsymbol{\mu}, \boldsymbol{\nu})\right)$ is achievable in the OMAC using TG inputs, where $\boldsymbol{\mu}=\left(\mu_{1}, \mu_{2}\right)$ and $\boldsymbol{\nu}=\left(\nu_{1}, \nu_{2}\right)$ are in $\mathbb{R}^{2}$ so that $\tilde{\mu}_{i} \leq \mathcal{E}_{i}$, and $\mathcal{R}_{\mathrm{t}}(\boldsymbol{\mu}, \boldsymbol{\nu})$ is given by $\left(R_{1}, R_{2}\right) \in \mathbb{R}_{+}^{2}$ satisfying

$$
\begin{aligned}
R_{i} & \leq \frac{1}{2} \log \left(\frac{\nu_{i}^{2}}{\tilde{\nu}_{i}^{2}}+\frac{\nu_{i}^{2}}{\sigma^{2}}\right)-\phi_{i}, i \in\{1,2\}, \\
R_{1}+R_{2} & \leq \frac{1}{2} \log \left(\frac{\nu_{1}^{2} \nu_{2}^{2}}{\tilde{\nu}_{1}^{2} \widetilde{\nu}_{2}^{2}}+\frac{\nu_{1}^{2} \nu_{2}^{2}}{\sigma^{2} \widetilde{\nu}_{2}^{2}}+\frac{\nu_{1}^{2} \nu_{2}^{2}}{\tilde{\nu}_{1}^{2} \sigma^{2}}\right)-\phi_{1}-\phi_{2},
\end{aligned}
$$

with $\phi_{i}$ as defined in Lemma 2. Thus $\mathcal{C} \supseteq \mathcal{R}_{\mathrm{T}}$

Proof: According to (3), for $\left(R_{1}, R_{2}\right)$ to be achievable, $R_{1}$ must satisfy $R_{1} \leq I\left(X_{1} ; Y \mid X_{2}\right)$. Note that $I\left(X_{1} ; Y \mid X_{2}\right)=$ $I\left(X_{1} ; X_{1}+Z\right)$. By Lemma 2 , if $X_{1}$ is $\left(\mu_{1}, \nu_{1}, \mathcal{A}_{1}\right)$-TG distributed, then $I\left(X_{1} ; X_{1}+Z\right) \geq \frac{1}{2} \log \left(\frac{\nu_{1}^{2}}{\tilde{\nu}_{1}^{2}}+\frac{\nu_{1}^{2}}{\sigma^{2}}\right)-\phi_{1}$, which proves the achievability of (29). The rates must also satisfy $R_{1}+R_{2} \leq I\left(X_{1}, X_{2} ; Y\right)=I\left(X_{1} ; Y \mid X_{2}\right)+$ $I\left(X_{2} ; Y\right)$. But $I\left(X_{1} ; Y \mid X_{2}\right)$ is lower bounded as above by Lemma 2. Moreover, when $X_{1}$ and $X_{2}$ follow $\left(\mu_{1}, \nu_{1}, \mathcal{A}_{1}\right)$ TG and $\left(\mu_{2}, \nu_{2}, \mathcal{A}_{2}\right)$-TG distributions, then $I\left(X_{2} ; Y\right) \geq$ $\frac{1}{2} \log \left(\frac{\nu_{2}^{2}}{\tilde{\nu}_{2}^{2}}+\frac{\nu_{2}^{2}}{\tilde{\nu}_{1}^{2}+\sigma^{2}}\right)-\phi_{2}$ by Lemma 3. Therefore,

$$
\begin{aligned}
& I\left(X_{1}, X_{2} ; Y\right) \\
& \geq \frac{1}{2} \log \left(\frac{\nu_{1}^{2}}{\tilde{\nu}_{1}^{2}}+\frac{\nu_{1}^{2}}{\sigma^{2}}\right)+\frac{1}{2} \log \left(\frac{\nu_{2}^{2}}{\tilde{\nu}_{2}^{2}}+\frac{\nu_{2}^{2}}{\tilde{\nu}_{1}^{2}+\sigma^{2}}\right)-\phi_{1}-\phi_{2} \\
& =\frac{1}{2} \log \left(\frac{\nu_{1}^{2} \nu_{2}^{2}}{\tilde{\nu}_{1}^{2} \tilde{\nu}_{2}^{2}}+\frac{\nu_{1}^{2} \nu_{2}^{2}}{\sigma^{2} \tilde{\nu}_{2}^{2}}+\frac{\nu_{1}^{2} \nu_{2}^{2}}{\tilde{\nu}_{1}^{2} \sigma^{2}}\right)-\phi_{1}-\phi_{2} .
\end{aligned}
$$

The convex-hull of $\bigcup_{\boldsymbol{\mu}, \boldsymbol{\nu}} \mathcal{R}_{\mathrm{t}}(\boldsymbol{\mu}, \boldsymbol{\nu})$ is achieved by time-sharing between achievable rate pairs, which concludes the proof.

Good choices of $(\boldsymbol{\mu}, \boldsymbol{\nu})$ can be obtained as in [32], which leads to simpler $\mathcal{R}_{\mathrm{t}}(\boldsymbol{\mu}, \boldsymbol{\nu})$. One such choice is provided next.

2) Achievable Region Simplification: Choosing $\mu_{i} \in\left[0, \mathcal{A}_{i}\right]$ and $\nu_{i}=\frac{1}{3} \min \left\{\mu_{i}, \mathcal{A}_{i}-\mu_{i}\right\}$ simplifies the inner bound since the resulting $\left(\mu_{i}, \nu_{i}, \mathcal{A}_{i}\right)$-TG distribution becomes almost identical to a Gaussian distribution $g_{\mu_{i}, \nu_{i}}(x)$. We use this to simplify $\mathcal{R}_{\mathrm{t}}(\boldsymbol{\mu}, \boldsymbol{\nu})$ as follows.
Proposition 1: The region $\mathcal{R}_{\mathrm{T}}^{\prime}$ defined as the set of rate pairs $\left(R_{1}, R_{2}\right) \in \mathbb{R}_{+}^{2}$ satisfying

$$
\begin{aligned}
R_{i} & \leq\left[\frac{1}{2} \log \left(1+\frac{\alpha_{i}^{2} \mathcal{A}_{i}^{2}}{9 \sigma^{2}\left(1+\epsilon_{\mu}\right)^{2}}\right)-\epsilon_{\phi}\right]^{+}, i \in\{1,2\} \\
R_{1}+R_{2} & \leq\left[\frac{1}{2} \log \left(1+\frac{\alpha_{1}^{2} \mathcal{A}_{1}^{2}+\alpha_{2}^{2} \mathcal{A}_{2}^{2}}{9 \sigma^{2}\left(1+\epsilon_{\mu}\right)^{2}}\right)-2 \epsilon_{\phi}\right]^{+}
\end{aligned}
$$

is achievable, with $\epsilon_{\phi}=0.016$ and $\epsilon_{\mu}=0.0015$.

Proof: We fix $\mu_{i} \geq 0$ and $\nu_{i}=\frac{\mu_{i}}{3}$ so that $\tilde{\mu}_{i}=\alpha_{i} \mathcal{A}_{i}$. Then $\mu_{i} \leq \tilde{\mu}_{i} \leq \frac{\mathcal{A}_{i}}{2}, i=1,2$. This choice leads to $\phi_{i} \leq \epsilon_{\phi}$, $\mu_{i} \leq \tilde{\mu}_{i}<\mu_{i}\left(1+\epsilon_{\mu}\right)$, and $\left(1-\epsilon_{\nu}\right) \nu_{i}^{2} \leq \tilde{\nu}_{i}^{2} \leq \nu_{i}^{2}$, where $\epsilon_{\phi}=0.016, \epsilon_{\mu}=0.0015$, and $\epsilon_{\nu}=0.0267$ (see [22, Appendix A]). Thus, we can write

$$
\frac{1}{2} \log \left(\frac{\nu_{i}^{2}}{\tilde{\nu}_{i}^{2}}+\frac{\nu_{i}^{2}}{\sigma^{2}}\right)-\phi_{i} \geq \frac{1}{2} \log \left(1+\frac{\nu_{i}^{2}}{\sigma^{2}}\right)-\epsilon_{\phi},
$$

and

$$
\begin{gathered}
\frac{1}{2} \log \left(\frac{\nu_{1}^{2} \nu_{2}^{2}}{\tilde{\nu}_{1}^{2} \tilde{\nu}_{2}^{2}}+\frac{\nu_{1}^{2} \nu_{2}^{2}}{\sigma^{2} \widetilde{\nu}_{2}^{2}}+\frac{\nu_{1}^{2} \nu_{2}^{2}}{\tilde{\nu}_{1}^{2} \sigma^{2}}\right)-\phi_{1}-\phi_{2} \\
\geq \frac{1}{2} \log \left(1+\frac{\nu_{1}^{2}+\nu_{2}^{2}}{\sigma^{2}}\right)-2 \epsilon_{\phi} .
\end{gathered}
$$

Since the above selection leads to $\nu_{i}^{2} \geq \frac{\left(\alpha_{i} \mathcal{A}_{i}\right)^{2}}{9\left(1+\epsilon_{\mu}\right)^{2}}$, we obtain the statement of the proposition.

The main advantageous feature of $\mathcal{R}_{\mathrm{T}}^{\prime}$ is that it is a convex polytope. This will be helpful for deriving constant gap capacity approximations for the OMAC with high SNR as we shall see in Sec. V. At moderate and low SNR, a discrete input distribution similar to the one in [33] achieves better performance. This is described next.

\section{B. Discrete Input Distribution}

The optimal input distribution for an IM-DD P2P channel is shown to be discrete in [41]. This suggests that a discrete input distribution achieves good performance in the OMAC. Thus, we consider a discrete distribution on $X_{i}$ of the form

$$
p_{i}\left(x_{i}\right)=\sum_{k=0}^{K_{i}} a_{i k} \delta\left(x_{i}-k \ell_{i}\right), \quad i=1,2,
$$

for some $K_{i}$ and $\ell_{i}$ such that $K_{i} \ell_{i}=\mathcal{A}_{i}$ and $\mathbb{E}\left[X_{i}\right] \leq \mathcal{E}_{i}$. We choose the parameters of the distributions so that $k_{1} \ell_{1} \neq k_{2} \ell_{2}$ for all $k_{i} \in\left\{1, \ldots, K_{i}\right\}, i=1,2$. This guarantees that no overlap occurs between symbols of $X_{1}$ and $X_{2}$ at the receiver, i.e., $X_{1}+X_{2}$ has $\left(K_{1}+1\right)\left(K_{2}+1\right)$ possibilities. ${ }^{6}$

1) Achievable Rate Region: To enlarge the achievable rate region, we need to optimize the input distribution. To this end, we focus on successive decoding where the receiver decodes $X_{1}$ first followed by $X_{2}$. The achievable rates are given by $R_{1}=I\left(X_{1} ; Y\right)$ and $R_{2}=I\left(X_{2} ; Y \mid X_{1}\right)$. We wish to maximize the two rates. We do this in two steps. This does not necessarily lead to the optimal distribution, but to one with good performance nevertheless.

\footnotetext{
${ }^{6}$ If $\mathcal{A}_{1}=\mathcal{A}_{2}$, then $k_{1} \ell_{1} \neq k_{2} \ell_{2}$ for all $k_{i} \in\left\{1, \ldots, K_{i}\right\}$ can not be guaranteed since $\mathcal{A}_{1}=K_{1} \ell_{1}=K_{2} \ell_{2}=\mathcal{A}_{2}$. This can be resolved by slightly reducing $\mathcal{A}_{2}$.
} 
We start by considering $I\left(X_{2} ; Y \mid X_{1}\right)=h\left(X_{2}+Z\right)-$ $h(Z) \geq \frac{1}{2} \log \left(\frac{e^{2 H\left(X_{2}\right)}}{2 \pi e \sigma^{2}}+1\right)$ which follows from the entropy power inequality. We aim for maximizing $H\left(X_{2}\right)$ as in [33] which eventually increases $I\left(X_{2} ; Y \mid X_{1}\right)$. The difference between the IM-DD P2P channel [33] and the OMAC appears in the next step. We need to maximize $I\left(X_{1} ; Y\right)$ where $Y$ includes the desired signal $X_{1}$ plus the sum of a discrete-distributed interference $X_{2}$ and a Gaussian-distributed noise $Z$. To maximize this term, we fix $H\left(X_{2}\right)$, and we consider $I\left(X_{1} ; Y\right)=h\left(X_{1}+X_{2}+Z\right)-h\left(X_{2}+Z\right) \geq$ $\frac{1}{2} \log \left(e^{2 H\left(X_{1}+X_{2}\right)}+2 \pi e \sigma^{2}\right)-h\left(X_{2}+Z\right)$. Note that $h\left(X_{2}+Z\right)$ is now fixed. To increase $I\left(X_{1} ; Y\right)$, we maximize $H\left(X_{1}+X_{2}\right)$. But since $k_{1} \ell_{1} \neq k_{2} \ell_{2}$ for all $k_{i} \in\left\{1, \ldots, K_{i}\right\}, i=1,2$, we can write $H\left(X_{1}+X_{2}\right)=H\left(X_{1}\right)+H\left(X_{2}\right)$. Since $H\left(X_{2}\right)$ is fixed now, it remains to maximize $H\left(X_{1}\right)$.

After this treatment, the maximization problems can be written for $i \in\{1,2\}$ as

$$
\begin{array}{rl}
\max _{a_{i k}} & H\left(X_{i}\right) \\
\text { s.t. } & \sum_{k=0}^{K_{i}} a_{i k}=1, \quad \sum_{k=0}^{K_{i}} a_{i k} k \ell_{i}=\mathcal{E}_{i}, \quad K_{i} \ell_{i}=\mathcal{A}_{i} .
\end{array}
$$

The solution of this problem is given by [33]

$$
a_{i k}=\frac{t_{i}^{k}}{\sum_{j=0}^{K_{i}} t_{i}^{j}}, \quad k=0, \ldots, K_{i},
$$

where $t_{i} \in[0,1]$ is the solution of $\sum_{k=0}^{K_{i}}\left(1-\frac{k \mathcal{A}_{i}}{K_{i} \mathcal{E}_{i}}\right) t_{i}^{k}=0$. This $t_{i}$ exists if $\mathcal{E}_{i} \leq \frac{\mathcal{A}_{i}}{2}$, which is guaranteed since $\alpha_{i} \leq \frac{1}{2}$. Note that the same distributions can be used for the other decoding order.

The achievable rate region can be simply obtained by plugging the obtained distributions in (3), and maximizing with respect to $K_{i}$ as described next.

Definition 1: Denote by $p_{i}^{\left[K_{i}\right]}\left(x_{i}\right)$ the distribution of $X_{i} \in$ $\left[0, \mathcal{A}_{i}\right]$ with $K_{i}+1$ mass points satisfying $\mathbb{E}\left[X_{i}\right]=\mathcal{E}_{i}$ obtained from (34) and (37), $i \in\{1,2\}$.

Proposition 2: The rate region $\mathcal{R}_{\mathrm{D}}=$ $\mathrm{CH}\left(\bigcup_{K_{1}, K_{2}} \mathcal{R}_{\mathrm{d}}\left(K_{1}, K_{2}\right)\right)$ is achievable in the OMAC, where $\mathcal{R}_{\mathrm{d}}\left(K_{1}, K_{2}\right)$ is the set of rate pairs $\left(R_{1}, R_{2}\right) \in \mathbb{R}_{+}^{2}$ satisfying (3) with $X_{i}, i \in\{1,2\}$, distributed according to $p_{i}^{\left[K_{i}\right]}\left(x_{i}\right)$.

Proof: Follows from the capacity of the MAC [30], [31] as given in (3).

2) Simplification at Low SNR: At low SNR, it suffices to choose $K_{1}=K_{2}=1$, i.e., each transmitter uses on-off keying (OOK). The achievable rates can also be simplified in this case as provided next.

Theorem 3: At low SNR, the achievable rate region $\mathcal{R}_{\mathrm{d}}(1,1)$ asymptotically coincides with the region described by

$$
0 \leq R_{1} \leq \frac{\alpha_{1}\left(1-\alpha_{1}\right) \mathcal{A}_{1}^{2}}{2 \sigma^{2}} \text {, and } 0 \leq R_{2} \leq \frac{\alpha_{2}\left(1-\alpha_{2}\right) \mathcal{A}_{2}^{2}}{2 \sigma^{2}} \text {. }
$$

Proof: A rate $R_{1}$ is achievable if $R_{1} \leq I\left(X_{1} ; Y \mid X_{2}\right)=$ $I\left(X_{1} ; X_{1}+Z\right)$. It was shown in [42] that this mutual information can be expressed as $\frac{\operatorname{var}\left(X_{1}\right)}{2 \sigma^{2}}+o\left(\mathcal{A}_{1}^{2}\right)$ where $\frac{o\left(\mathcal{A}_{1}^{2}\right)}{\mathcal{A}_{1}^{2}} \rightarrow 0$ as $\mathcal{A}_{1} \rightarrow 0$ and $\operatorname{var}\left(X_{1}\right)$ is the variance of $X_{1}$. Let $X_{1}$ be distributed according to $p_{1}^{[1]}\left(x_{1}\right)$. This leads to $\operatorname{var}\left(X_{1}\right)=$ $\alpha_{1}\left(1-\alpha_{1}\right) \mathcal{A}_{1}^{2}$, which leads to the first bound. The second follows similarly by choosing $X_{2}$ distributed according to $p_{2}^{[1]}\left(x_{2}\right)$. Finally, for $\left(R_{1}, R_{2}\right)$ to be achievable, it is required that $R_{1}+R_{2} \leq I\left(X_{1}, X_{2} ; Y\right)=I\left(X_{1}+X_{2} ; Y\right)$. With the above binary distributions, $X=X_{1}+X_{2}$ has variance $\operatorname{var}(X)=\alpha_{1}\left(1-\alpha_{1}\right) \mathcal{A}_{1}^{2}+\alpha_{2}\left(1-\alpha_{2}\right) \mathcal{A}_{2}^{2}$. Thus, this rate constraint becomes redundant at low SNR leading to the statement of the theorem.

Now that we have the achievable rate regions of the two distributions when successive cancellation decoding is used, it remains to express the achievable rate of the same distributions when time-division multiple-access (TDMA) is used instead. These expressions are useful in order to see how much performance loss does TDMA incur in comparison to successive cancellation decoding and to the capacity outer bounds.

\section{Time-Division Multiple-Access}

TDMA is a convenient strategy in practice due to its simplicity. We thus include it in our comparison to assess its performance. We distinguish between two variants: simple TDMA (s-tdma) and TDMA with intensity control (c-tdma). In the first variant, transmitter $i$ sends with average intensity $\mathcal{E}_{i}$ during a fraction $\tau_{i}$ of time, with $\tau_{1}+\tau_{2}=1$. In TDMA with intensity control, transmitter $i$ sends at an average intensity of $\min \left\{\frac{\mathcal{E}_{i}}{\tau_{i}}, \frac{\mathcal{A}_{i}}{2}\right\}$. The following simple corollaries provide the achievable rates with TDMA.

Corollary 2: Simple TDMA achieves the set of rate pairs $\mathcal{R}_{\mathrm{Q}}^{\text {s-tdma }}, \mathrm{Q} \in\{\mathrm{T}, \mathrm{D}\}$ (TG and Discrete, respectively), defined by $\left(R_{1}, R_{2}\right) \in \mathbb{R}_{+}^{2}$ satisfying $\frac{R_{1}}{R_{1, Q}}+\frac{R_{2}}{R_{2, Q}}=1$, where

$$
\begin{aligned}
\bar{R}_{i, \mathrm{~T}} & =\max _{\mu_{i}, \nu_{i}: \tilde{\mu}_{i}=\mathcal{E}_{i}} \frac{1}{2} \log \left(\frac{\nu_{i}^{2}}{\tilde{\nu}_{i}^{2}}+\frac{\nu_{i}^{2}}{\sigma^{2}}\right)-\phi_{i}, \\
\bar{R}_{i, \mathrm{D}} & =\max _{K_{i}} I\left(X_{i} ; X_{i}+Z\right), \quad X_{i} \sim p_{i}^{\left[K_{i}\right]}\left(x_{i}\right) .
\end{aligned}
$$

for $i \in\{1,2\}$ and $\tilde{\mu}_{i}, \tilde{\nu}_{i}$, and $p_{i}^{\left[K_{i}\right]}\left(x_{i}\right)$ are as described in (22), (23), and Def. 1, respectively.

Corollary 3: TDMA with intensity control achieves the region $\mathcal{R}_{\mathrm{Q}}^{\mathrm{c} \text {-tdma }}=\mathrm{CH}\left(\bigcup_{\tau_{1}, \tau_{2}} \mathcal{R}_{0, \mathrm{Q}}\left(\tau_{1}, \tau_{2}\right)\right), \mathrm{Q} \in\{\mathrm{R}, \mathrm{D}\}$, where $\tau_{1}, \tau_{2} \geq 0, \tau_{1}+\tau_{2}=1, \mathcal{R}_{0, Q}\left(\tau_{1}, \tau_{2}\right)$ is the set of $\left(R_{1}, R_{2}\right) \in \mathbb{R}_{+}^{2}$ satisfying $R_{i} \leq \tau_{i} \bar{R}_{i, \mathrm{Q}}, i \in\{1,2\}$ with $\bar{R}_{i, \mathrm{Q}}$ as defined in (38) with $\mathcal{E}_{i}$ replaced by $\min \left\{\frac{\mathcal{E}_{i}}{\tau_{i}}, \frac{\mathcal{A}_{i}}{2}\right\}$.

Next, we compare the established bounds. We focus on high SNR first, then low SNR, and finally moderate SNR.

\section{COMPARISON AND CAPACITY RESUlTS}

At high SNR, the derived bounds leads to a capacity approximation within a constant gap as described next.

\section{A. High SNR Capacity Approximation}

At high SNR, the outer bounds $\overline{\mathcal{C}}^{[1]}$ and $\overline{\mathcal{C}}^{[2]}$ in addition to the inner bound $\mathcal{R}_{\mathrm{T}}$ achieved with TG inputs provide a satisfactory bounding of $\mathcal{C}$. Fig. 3 shows the outer bound in Theorem 1, the asymptotic region in Corollary 1 , and the TG inner bound in Theorem 2 at high SNR for $\alpha_{1}=\alpha_{2}=\alpha$ 


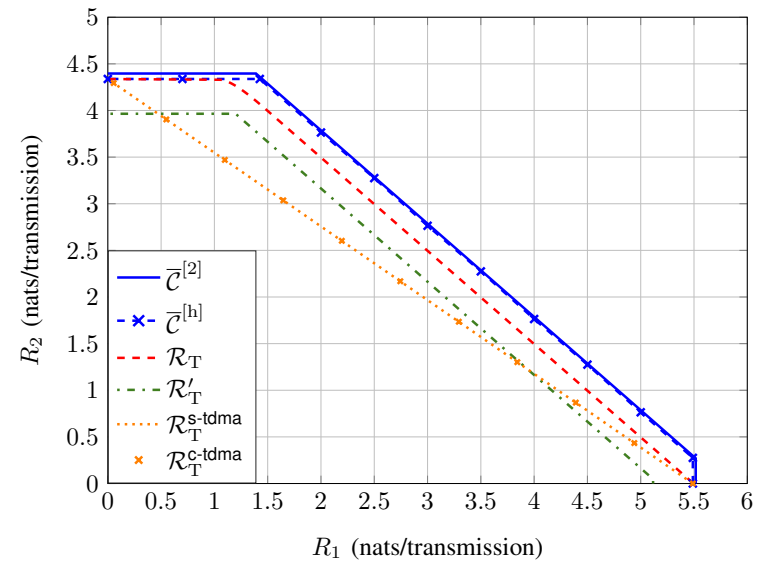

(a) $\alpha_{1}=\alpha_{2}=\frac{1}{2}$.

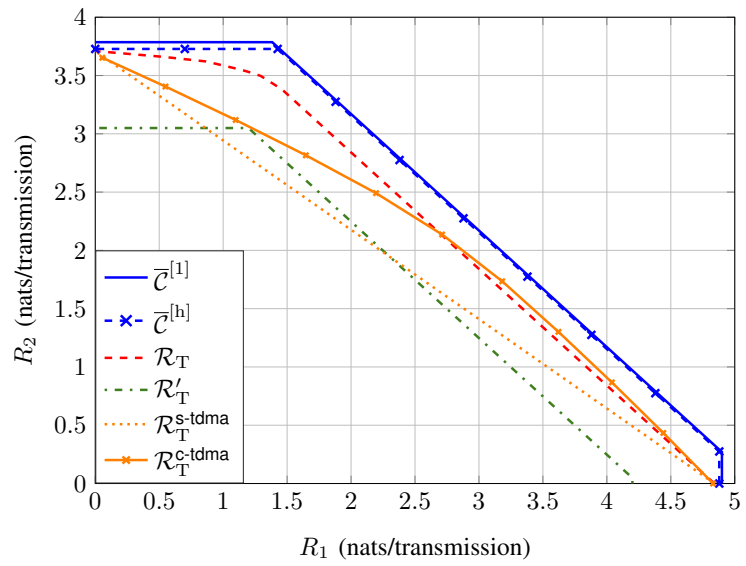

(b) $\alpha_{1}=\alpha_{2}=\frac{1}{5}$.

Fig. 3: Capacity region outer and inner bounds for an OMAC with $\frac{\mathcal{A}_{1}}{\sigma}=30 \mathrm{~dB}$ and $\frac{\mathcal{A}_{2}}{\sigma}=25 \mathrm{~dB}$.

under two cases: $\alpha=\frac{1}{2}$ and $\alpha=\frac{1}{5}$. The inner bound $\mathcal{R}_{\mathrm{D}}$ is not plotted here because it nearly coincides with $\mathcal{R}_{\mathrm{T}}$. Note that for $\alpha=\frac{1}{2}$, TDMA with intensity control coincides with simple TDMA because $\mathcal{E}_{i}=\frac{\mathcal{A}_{i}}{2}$ and hence $\min \left\{\frac{\mathcal{E}_{i}}{\tau_{i}}, \frac{\mathcal{A}_{i}}{2}\right\}=\frac{\mathcal{A}_{i}}{2}$ in this case. However, if $\alpha<\frac{1}{2}$, then TDMA with intensity control improves upon simple TDMA, and even achieves rates outside $\mathcal{R}_{\mathrm{T}}$. TDMA achieves appreciable performance in this case.

The high SNR asymptotic region in Corollary 1 nearly coincides with $\overline{\mathcal{C}}^{[j]}, j \in\{1,2\}$, in this case. Moreover, the inner bounds $\mathcal{R}_{\mathrm{T}}$ is fairly close to this outer bound. The inner bound $\mathcal{R}_{\mathrm{T}}^{\prime}$ serves as means to bound the gap between the TG inner bound an the outer bounds, owing to its simple expression as shown next.

Corollary 4: For the OMAC with 2 transmitters, the following statements hold asymptotically at high SNR:

1) The capacity region $\mathcal{C}$ and the region $\overline{\mathcal{C}}^{\text {[h] }}$ are within a gap of at most $\delta=\frac{1}{2} \log \left(\frac{9 e}{2 \pi}\right)$ nats/transmission. ${ }^{7}$

2) The symmetric capacity $C_{\mathrm{s}}$ and $\bar{C}_{\mathrm{s}}^{[\mathrm{h}]} \triangleq$ $\frac{1}{4} \log \left(1+\frac{c\left(\alpha_{12}\right)\left(\mathcal{A}_{1}+\mathcal{A}_{2}\right)^{2}}{\sigma^{2}}\right)$ are within a gap of at most $\delta_{\mathrm{s}}=\frac{1}{2} \log \left(\frac{3 \sqrt{e}}{\sqrt{\pi}}\right)$ nats/transmission.

Proof: We start by subtracting the rate constraints describing the region $\overline{\mathcal{C}}^{[\mathrm{h}]}$ (Corollary 1) and the inner bound $\mathcal{R}_{\mathrm{T}}^{\prime}$ (Proposition 1), we note that the gap between the constraints on $R_{i}, i \in\{1,2\}$, satisfy

$$
\frac{1}{2} \log \left(\frac{9 c\left(\alpha_{i}\right)}{\alpha_{i}^{2}}\right) \leq \frac{1}{2} \log \left(\frac{9 e}{2 \pi}\right)=\delta,
$$

nats/transmission (we neglect $\epsilon_{\mu}$ and $\epsilon_{\phi}$ at high SNR). On the other hand, the gap between the constraints on $R_{1}+R_{2}$ satisfies

$$
\begin{aligned}
\frac{1}{2} \log \left(\frac{9 c\left(\alpha_{12}\right)\left(\mathcal{A}_{1}+\mathcal{A}_{2}\right)^{2}}{\alpha_{1}^{2} \mathcal{A}_{1}^{2}+\alpha_{2}^{2} \mathcal{A}_{2}^{2}}\right) & \leq \frac{1}{2} \log \left(\frac{9 e \alpha_{12}^{2}\left(\mathcal{A}_{1}+\mathcal{A}_{2}\right)^{2}}{2 \pi\left(\alpha_{1}^{2} \mathcal{A}_{1}^{2}+\alpha_{2}^{2} \mathcal{A}_{2}^{2}\right)}\right) \\
\leq & \frac{1}{2} \log \left(\frac{9 e}{\pi}\right)
\end{aligned}
$$

\footnotetext{
${ }^{7}$ We say that two regions $\mathcal{R}_{1}$ and $\mathcal{R}_{2}$ are within a gap of $\delta$ if $\left(R_{1}, R_{2}\right) \in$ $\mathcal{R}_{1}$ implies that $\left(R_{1}+\delta, R_{2}+\delta\right) \notin \mathcal{R}_{2}$.
}

nats/transmission by definition of $\alpha_{12}$ (Theorem 1) and using the inequality $\left(\alpha_{1} \mathcal{A}_{1}+\alpha_{2} \mathcal{A}_{2}\right)^{2} \leq 2 \alpha_{1}^{2} \mathcal{A}_{1}^{2}+2 \alpha_{2}^{2} \mathcal{A}_{2}^{2}$. This leads to a gap of at most $\frac{1}{2} \log \left(\frac{3 \sqrt{e}}{\sqrt{\pi}}\right)<\delta$ nats/dimension. Since the outer bound $\overline{\mathcal{C}}^{[12]}$ given in Corollary 1 converges to $\overline{\mathcal{C}}^{[\mathrm{h}]}$ as SNR increases, then the maximum gap per dimension between $\mathcal{R}_{\mathrm{T}}^{\prime}$ and $\overline{\mathcal{C}}^{[12]}$ also converges to a quantity upper bounded by $\delta$, which proves the first statement. The symmetric capacity statement follows similarly by noting that $\overline{\mathcal{C}}^{[12]}$ and $\overline{\mathcal{C}}^{\text {h] }}$ converge as SNR increases, and then by finding the intersections of the line $R_{1}=R_{2}$ with the boundary of the regions $\overline{\mathcal{C}}^{[\mathrm{h}]}$ and $\mathcal{R}_{\mathrm{T}}^{\prime}$. The intersection with $\overline{\mathcal{C}}^{[\mathrm{h}]}$ lies at the point $\left(R_{1}, R_{2}\right)$ satisfying $R_{1}=R_{2}=\bar{C}_{\mathrm{s}}^{[\mathrm{h}]}$. The intersection with $\mathcal{R}_{\mathrm{T}}^{\prime}$ lies at $\left(R_{1}, R_{2}\right)$ whose components converge $R_{1}=R_{2}=\frac{1}{4} \log \left(\frac{\alpha_{1}^{2} \mathcal{A}_{1}^{2}+\alpha_{2}^{2} \mathcal{A}_{2}^{2}}{9 \sigma^{2}}\right)$ as SNR increases (again neglecting $\epsilon_{\mu}$ and $\epsilon_{\phi}$ ). The gap $\delta_{\mathrm{S}}$ follows by upper bounding the difference between the two, which concludes the proof.

Note that the gap given in this corollary is negligible at high SNR relative to the rates which themselves become large at high SNR.

Thus, the TG input distribution can be used to construct codes that achieve capacity within a constant gap at high SNR. The performance of any other coding scheme can be compared against $\overline{\mathcal{C}}^{[\mathrm{h}]}$ to assess its performance with respect to the information-theoretic capacity.

\section{B. Low SNR Capacity Region}

At low SNR, the outer bound $\overline{\mathcal{C}}^{[3]}$ and the inner bound $\mathcal{R}_{\mathrm{D}}$ achieved with the discrete input distribution introduced in Definition 1 coincide as shown in Fig. 4. This figure also shows the achievable rate regions by using TDMA $\mathcal{R}_{\mathrm{D}}^{\text {s-tdma }}$ and $\mathcal{R}_{\mathrm{D}}^{\text {c-tdma }}$ for comparison. TDMA is outperformed by successive cancellation decoding here.

It turns out that at low SNR, it suffice to set $K_{1}=K_{2}=1$, i.e., the transmitters use on-off keying (OOK). This achieves the low-SNR capacity as given in the following theorem.

Theorem 4: For an OMAC with 2 transmitters, as SNR decreases, we have 


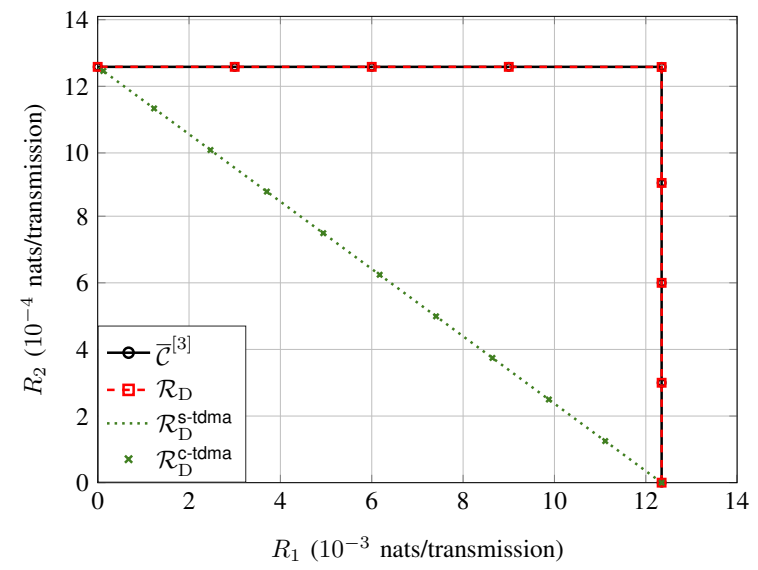

(a) $\alpha_{1}=\alpha_{2}=\frac{1}{2}$.

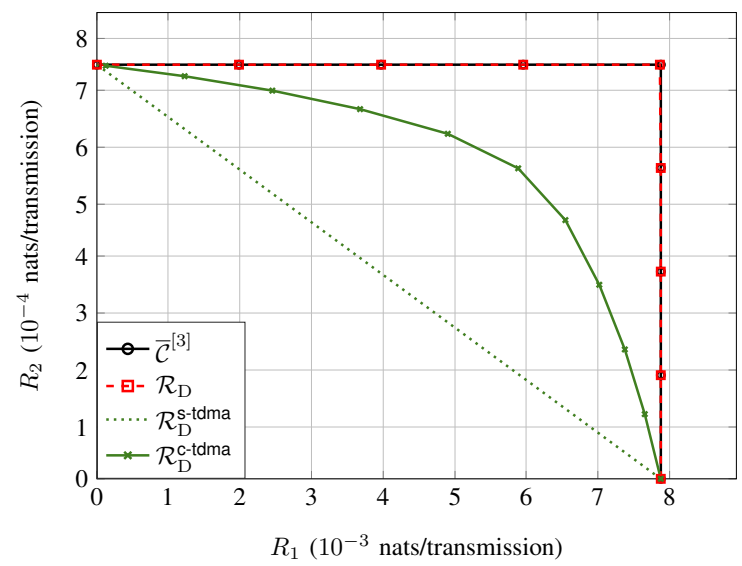

(b) $\alpha_{1}=\alpha_{2}=\frac{1}{5}$.

Fig. 4: Capacity region outer and inner bounds for an OMAC with $\frac{\mathcal{A}_{1}}{\sigma}=-5 \mathrm{~dB}$ and $\frac{\mathcal{A}_{2}}{\sigma}=-10 \mathrm{~dB}$.

1) $\mathcal{C} \doteq \mathcal{R}_{\mathrm{d}}(1,1)$ given in Theorem 3 . $^{8}$

2) $C_{\mathrm{s}} \doteq C_{\mathrm{s}}^{[1]} \triangleq \frac{1}{2 \sigma^{2}} \min _{i \in\{1,2\}} \alpha_{i}\left(1-\alpha_{i}\right) \mathcal{A}_{i}^{2}$.

Proof: The outer bound $\overline{\mathcal{C}}^{[3]}$ converges at low SNR to the region

$$
\begin{array}{r}
R_{i} \leq \frac{\alpha_{i}\left(1-\alpha_{i}\right) \mathcal{A}_{i}^{2}}{2 \sigma^{2}}, i \in\{1,2\}, \\
R_{1}+R_{2} \leq \frac{\alpha_{12}\left(1-\alpha_{12}\right)\left(\mathcal{A}_{1}+\mathcal{A}_{2}\right)^{2}}{2 \sigma^{2}} .
\end{array}
$$

Recall that $\alpha_{23}=\frac{\mathcal{E}_{1}+\mathcal{E}_{2}}{\mathcal{A}_{1}+\mathcal{A}_{2}}$. Note that the last constraint is redundant since $\alpha_{12}\left(1-\alpha_{12}\right)\left(\mathcal{A}_{1}+\mathcal{A}_{2}\right)^{2}>\alpha_{1}\left(1-\alpha_{1}\right) \mathcal{A}_{1}^{2}+$ $\alpha_{2}\left(1-\alpha_{2}\right) \mathcal{A}_{2}^{2}$. Thus, this outer bound coincides with the inner bound $\mathcal{R}_{\mathrm{d}}(1,1)$ at low SNR (cf. Theorem 3 ), which proves the statement of the theorem. The asymptotic symmetric capacity follows directly from the capacity region.

Therefore, based on this theorem, OOK combined with successive cancellation decoding is the optimal scheme at low SNR. The region $\mathcal{R}_{\mathrm{d}}(1,1)$ provides and information-theoretic benchmark that can be used to assess the performance of any coding scheme at low SNR.

\section{Moderate SNR}

As SNR increases from low to high SNR, the outer bound interchange their roles with $\overline{\mathcal{C}}^{[3]}$ becoming less tight and $\overline{\mathcal{C}}^{[1]}$ and $\overline{\mathcal{C}}^{[2]}$ becoming more tight. The inner bound $\mathcal{R}_{\mathrm{D}}$ preserves a good performance in the moderate SNR range as shown in Fig. 5. In this figure, we have computed the union in Proposition 2 for $K \in\{1, \ldots, 10\}$.

Note that a low value of $K$ suffices to achieve good performance at moderate SNR as shown in Fig. 6. Here, $\mathcal{R}_{\mathrm{d}}(1,1)$ defined in Proposition 2 corresponds to using a binary alphabet $(\mathrm{OOK}), \mathcal{R}_{\mathrm{d}}(2,2)$ corresponds to using a ternary one, and $\mathcal{R}_{\mathrm{d}}(3,3)$ corresponds to using a quaternary one. It can be seen that these inner bounds are fairly close to the outer bounds. Hence, the corresponding schemes are recommended in the moderate SNR regime.

\footnotetext{
${ }^{8} A \doteq B$ as $x$ decreases implies that $A$ and $B$ asymptotically coincide when $x \rightarrow 0$.
}

In the next section, the derived bounds and insights are extended to the $N$-transmitter OMAC.

\section{EXTENSION TO $N$ TRANSMITTERS}

The capacity region of the MAC with $N$ transmitters can be generally written as the convex-hull of the union over all permissible input distributions of the set of $\left(R_{1}, \ldots, R_{N}\right) \in$ $\mathbb{R}_{+}^{N}$ satisfying

$$
\sum_{i \in \mathcal{S}} R_{i}<I\left(X_{\mathcal{S}} ; Y \mid X_{\mathcal{S}^{c}}\right),
$$

for all $\mathcal{S} \subseteq \mathcal{N}$, where $\mathcal{S}^{c}$ is the complement of $\mathcal{S}$, and $X_{\mathcal{S}}=$ $\left(X_{s_{1}}, \ldots, X_{s_{|\mathcal{S}|}}\right)$ with $s_{j}, j \in\{1, \ldots,|\mathcal{S}|\}$, an element of $\mathcal{S}$. Based on this, the outer bounds in Theorem 1 and Corollary 1 can be written for the OMAC with $N$ transmitters as follows.

Theorem 5: The capacity region $\mathcal{C}$ of the OMAC with $N$ transmitters satisfies $\mathcal{C} \subseteq \overline{\mathcal{C}}^{[j]}, j \in\{1,2,3\}$, defined as the set of $\left(R_{1}, \ldots, R_{N}\right) \in \mathbb{R}_{+}^{N}$ satisfying $\sum_{i \in \mathcal{S}} R_{i} \leq$ $\bar{C}_{\alpha_{\mathcal{S}}}^{[j]}\left(\sum_{i \in \mathcal{S}} \mathcal{A}_{i}, \sigma\right)$ for all $\mathcal{S} \subseteq \mathcal{N}$, with

$$
\alpha_{\mathcal{S}}=\frac{\sum_{i \in \mathcal{S}} \mathcal{E}_{i}}{\sum_{i \in \mathcal{S}} \mathcal{A}_{i}}
$$

Based on this, we can write the following results for the high and low SNR regimes.

\section{A. High SNR}

A rate region which coincides with capacity outer bounds at high SNR is given in the following.

Corollary 5: At high SNR, the capacity region outer bound $\overline{\mathcal{C}}^{[1]} \cap \overline{\mathcal{C}}^{[2]}$ (Theorem 5) converges to $\overline{\mathcal{C}}^{[\mathrm{h}]}$ defined as the set of $\left(R_{1}, \ldots, R_{n}\right) \in \mathbb{R}_{+}^{N}$ satisfying

$$
\sum_{i \in \mathcal{S}} R_{i} \leq \frac{1}{2} \log \left(1+\frac{c\left(\alpha_{\mathcal{S}}\right)\left(\sum_{i \in \mathcal{S}} \mathcal{A}_{i}\right)^{2}}{\sigma^{2}}\right),
$$

for all $\mathcal{S} \subseteq \mathcal{N}$ where $\alpha_{\mathcal{S}}$ is defined in (44), and $c(\cdot)$ is defined in (11).

The proofs are similar to those of Theorem 1 and Corollary 1. This asymptotic region is achievable within a constant gap 


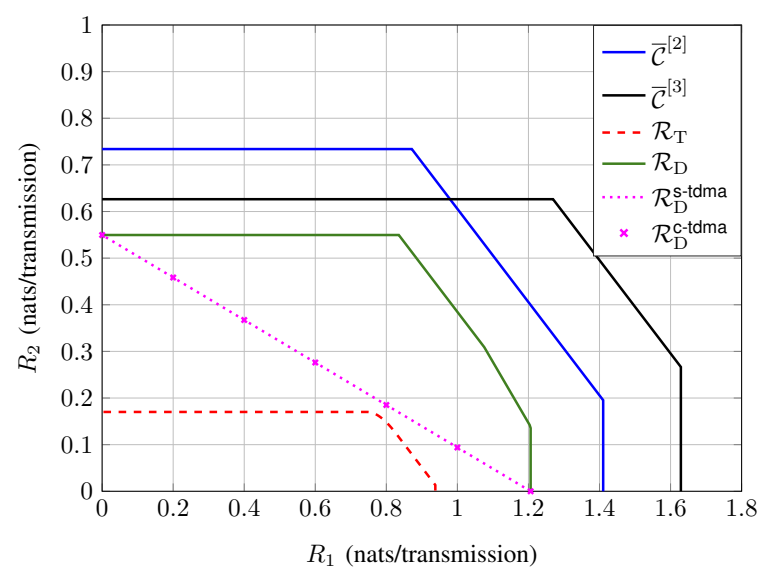

(a) $\alpha_{1}=\alpha_{2}=\frac{1}{2}$.

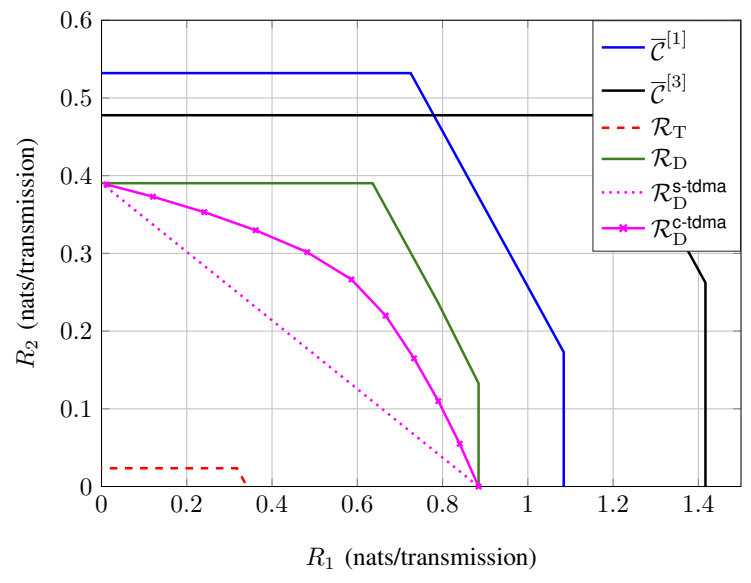

(b) $\alpha_{1}=\alpha_{2}=\frac{1}{5}$.

Fig. 5: Capacity region outer and inner bounds for an OMAC with $\frac{\mathcal{A}_{1}}{\sigma}=10 \mathrm{~dB}$ and $\frac{\mathcal{A}_{2}}{\sigma}=5 \mathrm{~dB}$.

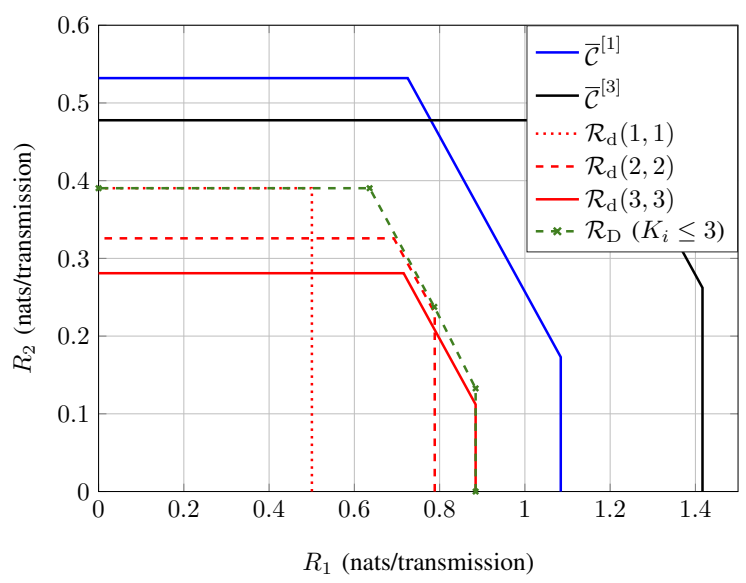

Fig. 6: Capacity region outer and inner bounds for an OMAC with $\alpha_{1}=\alpha_{2}=\frac{1}{5}, \frac{\mathcal{A}_{1}}{\sigma}=10 \mathrm{~dB}$ and $\frac{\mathcal{A}_{2}}{\sigma}=5 \mathrm{~dB}$.

at high SNR using a TG input distribution. Namely, using a TG input distribution, the following rate region is achievable.

Theorem 6: The rate region $\mathcal{R}_{\mathrm{T}}=\mathrm{CH}\left(\bigcup_{\mu, \nu} \mathcal{R}_{\mathrm{t}}(\boldsymbol{\mu}, \boldsymbol{\nu})\right)$ is achievable in the OMAC using TG inputs, where $\boldsymbol{\mu}=$ $\left(\mu_{1}, \ldots, \mu_{N}\right)$ and $\boldsymbol{\nu}=\left(\nu_{1}, \ldots, \nu_{N}\right)$ are in $\mathbb{R}^{2}$ so that $\tilde{\mu}_{i} \leq \mathcal{E}_{i}$, and $\mathcal{R}_{t}(\boldsymbol{\mu}, \boldsymbol{\nu})$ is given by $\left(R_{1}, \ldots, R_{N}\right) \in \mathbb{R}_{+}^{N}$ satisfying

$$
\sum_{i \in \mathcal{S}} R_{i} \leq \frac{1}{2} \log \left(\frac{\left(\sum_{i \in \mathcal{S}} \tilde{\nu}_{i}^{2}+\sigma^{2}\right) \prod_{i \in \mathcal{S}} \nu_{i}^{2}}{\prod_{i \in \mathcal{S}} \tilde{\nu}_{i}^{2}}\right)-\sum_{i \in \mathcal{S}} \phi_{i}
$$

for all $\mathcal{S} \subseteq \mathcal{N}$ with $\phi_{i}$ as defined in Lemma 2. Thus $\mathcal{C} \supseteq \mathcal{R}_{\mathrm{T}}$.

Proof: The proof of this theorem follows along similar lines as the proof of Theorem 2, but requires a simple extension of Lemma 3 to a channel with $Y=X+\sum_{j=1}^{J} W_{j}+Z$ where $W_{j}$ is $\left(\mu_{w_{j}}, \nu_{w_{j}}, \mathcal{A}_{w_{j}}\right)$-TG distributed and $Z \sim g_{0, \sigma}(z)$. This extension is given in Lemma 4 in Appendix B for completeness.

Similar to Proposition 1, a simplified region can be obtained using a specific choice of $\mu_{i}$ and $\nu_{i}$. The simplified region $\mathcal{R}_{\mathrm{T}}^{\prime}$ is given by the set of rate pairs $\left(R_{1}, \ldots, R_{N}\right) \in \mathbb{R}_{+}^{N}$ satisfying

$$
\sum_{i \in \mathcal{S}} R_{i} \leq \frac{1}{2} \log \left(1+\frac{\sum_{i \in \mathcal{S}} \alpha_{i}^{2} \mathcal{A}_{i}^{2}}{9 \sigma^{2}\left(1+\epsilon_{\mu}\right)^{2}}\right)-|\mathcal{S}| \epsilon_{\phi},
$$

for all $\mathcal{S} \subseteq \mathcal{N}$, where $\epsilon_{\phi}=0.016$ and $\epsilon_{\mu}=0.0015$.

The regions $\mathcal{R}_{\mathrm{T}}^{\prime}$ and $\overline{\mathcal{C}}^{[\mathrm{h}]}$ are within a constant gap at high SNR as given next.

Corollary 6: For an OMAC with $N$ transmitters, the following statements hold asymptotically at high SNR:

1) The capacity region $\mathcal{C}$ and the region $\overline{\mathcal{C}}^{[\mathrm{h}]}$ defined in Corollary 5 are within a gap of at most $\delta=\frac{1}{2} \log \left(\frac{9 e}{2 \pi}\right)$ nats/transmission.

2) The symmetric capacity $C_{\mathrm{s}}$ and $\bar{C}_{\mathrm{s}}^{[\mathrm{h}]}=$ $\frac{1}{2 N} \log \left(1+\frac{c\left(\alpha_{\mathcal{N}}\right)\left(\sum_{i=1}^{N} \mathcal{A}_{i}\right)^{2}}{\sigma^{2}}\right)$ are within a gap of at most $\delta_{\mathrm{s}}=\frac{1}{N} \log \left(\frac{3 \sqrt{e N}}{\sqrt{2 \pi}}\right)$ nats/transmission, where $\alpha_{\mathcal{N}}$ is as defined in (44).

Proof: The capacity region gap follows by comparing $\overline{\mathcal{C}}^{[\mathrm{h}]}$ and the inner bound $\mathcal{R}_{\mathrm{T}}^{\prime}$, and noting that the outer bound $\overline{\mathcal{C}}^{[1]} \cap$ $\overline{\mathcal{C}}^{[2]}$ converge to $\overline{\mathcal{C}}^{[\mathrm{h}]}$ as SNR increases. The gap between the constraints on $\sum_{i \in \mathcal{S}} R_{i}$ in these bounds at high SNR satisfies

$$
\begin{aligned}
\frac{1}{2} \log \left(\frac{9 c\left(\alpha_{\mathcal{S}}\right)\left(\sum_{i \in \mathcal{S}} \mathcal{A}_{i}\right)^{2}}{\sum_{i \in \mathcal{S}} \alpha_{i}^{2} \mathcal{A}_{i}^{2}}\right) & \leq \frac{1}{2} \log \left(\frac{9 e\left(\sum_{i \in \mathcal{S}} \alpha_{i} \mathcal{A}_{i}\right)^{2}}{2 \pi \sum_{i \in \mathcal{S}} \alpha_{i}^{2} \mathcal{A}_{i}^{2}}\right) \\
& \leq \frac{1}{2} \log \left(\frac{9 e|\mathcal{S}|}{2 \pi}\right),
\end{aligned}
$$

in nats/transmission. Thus, the gap per dimension is upper bounded by $\frac{1}{2|\mathcal{S}|} \log \left(\frac{9 e|\mathcal{S}|}{2 \pi}\right)<\delta$. To bound the symmetric capacity gap, we find the intersection of the boundary of the region $\overline{\mathcal{C}}^{[\mathrm{h}]}$ with the line $R_{1}=R_{2}=\cdots=R_{N}$. This intersection lies at $R_{1}=R_{2}=\cdots=R_{N}=$ $\bar{C}_{\mathrm{s}}^{[\mathrm{h}]}$. The achievable symmetric rate from (46) converges to $\frac{1}{2 N} \log \left(1+\frac{\sum_{i=1}^{N} \alpha_{i}^{2} \mathcal{A}_{i}^{2}}{9 \sigma^{2}}\right)$ as SNR increases. This leads to a gap of $\frac{1}{N} \log \left(\frac{3 \sqrt{e N}}{\sqrt{2 \pi}}\right)$ which concludes the proof.

Note that the symmetric capacity gap $\delta_{\mathrm{s}}$ vanishes as $N$ grows. The symmetric capacity upper and lower bounds 
are plotted in Fig. 7 for comparison. This figure shows that the gap between the symmetric rate obtained from $\mathcal{R}_{\mathrm{T}}^{\prime}$ and the symmetric capacity upper bound $\bar{C}_{\mathrm{s}}^{[\mathrm{h}]}$ are within $\delta_{\mathrm{s}}$ nats/transmission, which confirms Corollary 6 . Note that the symmetric rate obtained from $\mathcal{R}_{\mathrm{T}}$ is closer to the upper bound.

\section{B. Moderate and Low SNR}

In this case, the discrete input distribution achieves higher rates than the TG distribution. The corresponding achievable rate region is given as follows.

Proposition 3: The rate region $\mathcal{R}_{\mathrm{D}}=$ $\mathrm{CH}\left(\bigcup_{K_{1}, \ldots, K_{N}} \mathcal{R}_{\mathrm{d}}\left(K_{1}, \ldots, K_{N}\right)\right)$ is achievable in the OMAC, where $\mathcal{R}_{\mathrm{d}}\left(K_{1}, \ldots, K_{N}\right)$ is the set of rate pairs $\left(R_{1}, \ldots, R_{N}\right) \in \mathbb{R}_{+}^{N}$ satisfying (43) with $X_{i}, i \in \mathcal{N}$, distributed according to $p_{i}^{\left[K_{i}\right]}\left(x_{i}\right)$ given in Definition 1.

This achievable rate region coincides with capacity at low SNR, where it suffices to choose $K_{i}=1$ for all $i \in \mathcal{N}$. This leads to the following.

Theorem 7: For an OMAC with $N$ transmitters, as SNR decreases, we have

1) The region $\mathcal{R}_{\mathrm{d}}(1, \ldots, 1)$ converges to the set of $\left(R_{1}, \ldots, R_{N}\right) \in \mathbb{R}_{+}^{N}$ satisfying $0 \leq R_{i} \leq \frac{\alpha_{i}\left(1-\alpha_{i}\right) \mathcal{A}_{i}^{2}}{2 \sigma^{2}}$.

2) $\mathcal{C} \doteq \mathcal{R}_{\mathrm{d}}(1, \ldots, 1)$.

3) $C_{\mathrm{s}} \doteq C_{\mathrm{s}}^{[1]}=\frac{1}{2 \sigma^{2}} \min _{i \in \mathcal{N}} \alpha_{i}\left(1-\alpha_{i}\right) \mathcal{A}_{i}^{2}$.

Proof: The proof of the first statement is similar to Theorem 3. The low-SNR capacity and symmetric capacity statements follow from the asymptotic behavior of $\overline{\mathcal{C}}^{[3]}$ at low SNR as in Theorem 4.

The symmetric capacity bounds at low SNR are plotted in Fig. 7 for comparison for a system with three transmitters. This figure shows the convergence of the symmetric rates obtained from the outer bound $\overline{\mathcal{C}}^{[3]}$ and the inner bound $\mathcal{R}_{d}(1,1,1)$ at low SNR. It also shows that the low-SNR capacity is characterized by $C_{\mathrm{s}}^{[1]}$.

\section{CONCLUSION}

In this work, we have studied the capacity region of the intensity-modulation direct-detection (IM-DD) optical multiple-access channel modeled as a Gaussian channel with input-independent noise. Using results on the capacity of the IM-DD optical point-to-point channel given in [25], [32], [33], we derived capacity region outer bounds and achievable rate region for the multiple-access case. The bounds provide a capacity approximation within a constant gap at high SNR, and characterize the capacity region at low SNR. These results advocate using schemes which mimic a truncated-Gaussian input distribution in order to achieve satisfactory performance at high SNR. On the other hand, at moderate and low SNR, a discrete input distribution achieves better performance, even if the number of mass points is low. Particularly, on-off keying (OOK) with successive cancellation decoding is optimal at low SNR. This is good news for practical systems since OOK is a simple scheme from an implementation point of view. The results provide fundamental information-theoretic benchmarks for comparing the achievable rates of coding schemes for the IM-DD multiple-access channel, and assessing their optimality.

Finally, it remains to say that input-dependent noise can be incorporated by making the variance of the input-independent noise depend on the input average and peak intensity constraints as in [43]. Alternatively, it can be incorporated by using a Gaussian channel with input-dependent noise model similar to [37].

\section{APPENDIX A}

SUFFICIENCY OF $\alpha_{i} \in[0,1 / 2]$

The capacity region of the OMAC is evaluated for $\bar{X}_{i}=$ $\mathbb{E}\left[X_{i}\right] \leq \mathcal{E}_{i}$ which is in turn equal to $\alpha_{i} \mathcal{A}_{i}$. Now consider an OMAC with $\alpha_{i}>\frac{1}{2}$ and let the transmit signals satisfy $\bar{X}_{i} \geq$ $\frac{\mathcal{A}_{i}}{2}$. The output of the channel is $Y=X_{1}+X_{2}+Z$. Consider the modified output signal given by $\tilde{Y}=-Y+\mathcal{A}_{1}+\mathcal{A}_{2}$. This transformation does not change the achievable rate region. But $\tilde{Y}$ can be also written as $\tilde{X}_{1}+\tilde{X}_{2}+Z$ with $\tilde{X}_{i}=\mathcal{A}_{i}-X_{i}$. Therefore, the transmitters can replace $X_{i}$ by $\tilde{X}_{i}$ without any impact on the achievable rates. Note that since $\bar{X}_{i} \geq \frac{\mathcal{A}_{i}}{2}$, then $\mathbb{E}\left[\tilde{X}_{i}\right] \leq \frac{\mathcal{A}_{i}}{2}$. Thus, an OMAC with $\alpha_{i}>\frac{1}{2}$ can be replaced by one with $\alpha_{i}=\frac{1}{2}$ without any loss in capacity.

\section{APPENDIX B \\ EXTENSION OF LEMMA 3}

In this appendix, we extend Lemma 3 to the scenario where interference is the sum of multiple TG-distributed random variables. For this case, we have the following statement.

Lemma 4: Consider a channel $Y=X+\sum_{j=1}^{J} W_{j}+Z$, with independent $X, W_{j}$ and $Z$ so that $Z \sim g_{0, \sigma}(z)$, and $W_{j}$ is $\left(\mu_{w_{j}}, \nu_{w_{j}}, \mathcal{A}_{w_{j}}\right)$-TG distributed. A $(\mu, \nu, \mathcal{A})$-TG distributed $X$ with $\tilde{\mu} \leq \mathcal{E}$ achieves a rate

$$
R=I(X ; Y) \geq \frac{1}{2} \log \left(\frac{\nu^{2}}{\tilde{\nu}^{2}}+\frac{\nu^{2}}{\sum_{j=1}^{J} \tilde{\nu}_{w_{j}}^{2}+\sigma^{2}}\right)-\phi,
$$

where $\phi$ is as in Lemma 2, where $\tilde{\nu}^{2}$ and $\tilde{\nu}_{w_{j}}^{2}$ are the variances of $X$ and $W_{j}$, respectively.

Proof: The achievable rate is given by

$$
R=I(X ; Y)=h(X)-h(X \mid Y) .
$$

Since $X$ is TG distributed, its entropy is given as

$$
h(X)=\frac{1}{2} \log \left(2 \pi e \nu^{2}\right)-\phi .
$$

Next, consider $h(X \mid Y)$. The covariance matrix of $(X, Y)$ is given by $\boldsymbol{S}=\left[\begin{array}{ll}\tilde{\nu}^{2} & \tilde{\nu}^{2} \\ \tilde{\nu}^{2} & \tilde{\nu}^{2}+\sum_{j=1}^{J} \tilde{\nu}_{w_{j}}^{2}+\sigma^{2}\end{array}\right]$. Under this covariance constraint, the conditional differential entropy $h(X \mid Y)$ is maximized by jointly Gaussian $(X, Y)$ [40]. This leads to

$$
\begin{aligned}
h(X \mid Y) & \leq \frac{1}{2} \log \left(\frac{2 \pi e|\boldsymbol{S}|}{\operatorname{var}(Y)}\right) \\
& =\frac{1}{2} \log \left(2 \pi e \frac{\tilde{\nu}^{2}\left(\sum_{j=1}^{J} \tilde{\nu}_{w_{j}}^{2}+\sigma^{2}\right)}{\tilde{\nu}^{2}+\sum_{j=1}^{J} \tilde{\nu}_{w_{j}}^{2}+\sigma^{2}}\right) .
\end{aligned}
$$




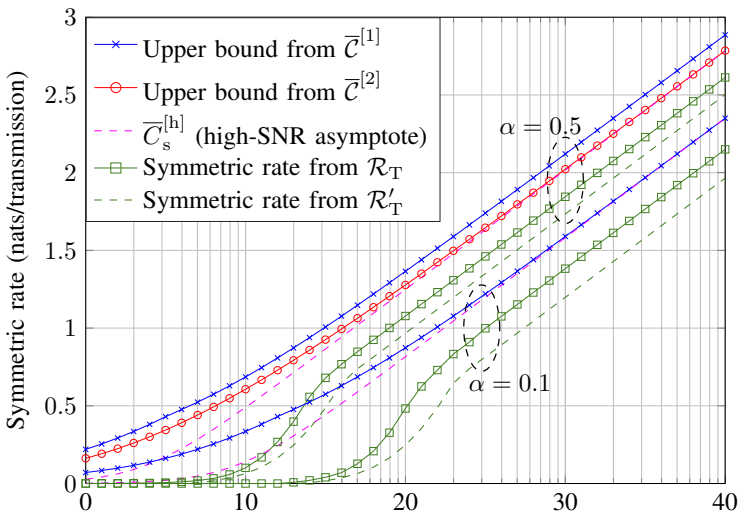

$\mathcal{A}_{1} / \sigma(\mathrm{dB})$

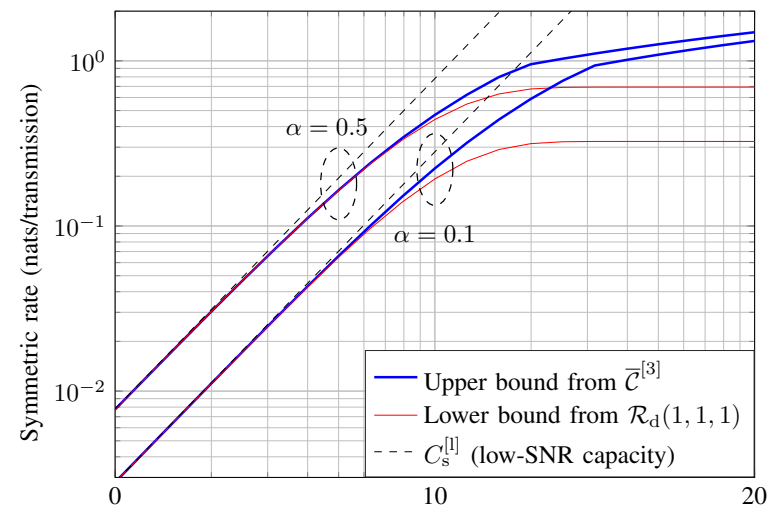

$\mathcal{A}_{1} / \sigma(\mathrm{dB})$

Fig. 7: Symmetric capacity bounds for an OMAC with $N=3, \mathcal{A}_{2}=\mathcal{A}_{1} / 2, \mathcal{A}_{3}=\mathcal{A}_{2} / 2$, and $\alpha_{1}=\alpha_{2}=\alpha_{3}=\alpha$.

Therefore, we have that

$$
\begin{aligned}
R & \geq \frac{1}{2} \log \left(2 \pi e \nu^{2}\right)-\phi+\frac{1}{2} \log \left(\frac{\tilde{\nu}^{2}+\sum_{j=1}^{J} \tilde{\nu}_{w_{j}}^{2}+\sigma^{2}}{2 \pi e \tilde{\nu}^{2}\left(\sum_{j=1}^{J} \tilde{\nu}_{w_{j}}^{2}+\sigma^{2}\right)}\right) \\
& =\frac{1}{2} \log \left(\frac{\nu^{2}}{\tilde{\nu}^{2}}+\frac{\nu^{2}}{\sum_{j=1}^{J} \tilde{\nu}_{w_{j}}^{2}+\sigma^{2}}\right)-\phi .
\end{aligned}
$$

This concludes the proof.

\section{REFERENCES}

[1] O. M. S. Al-Ebraheemy, A. Chaaban, T. Y. Al-Naffouri, and M. S. Alouini, "Capacity bounds for the 2-user Gaussian IM-DD optical multiple-access channel," in Proc. IEEE Int. Symp. Circuits Syst. (ISCAS), Montreal, Canada, May. 2016, pp. 2823-2826.

[2] M. A. Khalighi and M. Uysal, "Survey on free space optical communications: A communication theory perspective," Commun. Surveys Tuts, vol. 16, no. 4, pp. 2231-2258, 4th quarter 2014.

[3] D. K. Borah, A. C. Boucouvalas, C. C. Davis, S. Hranilovic, and K. Yiannopoulos, "A review of communication-oriented optical wireless systems," EURASIP J. Wireless Commun. Netw., vol. 2012, no. 1, pp. $1-28,2012$.

[4] H. Elgala, R. Mesleh, and H. Haas, "Indoor optical wireless communication: Potential and state-of-the-art," IEEE Comm. Magazine, vol. 49, no. 9, pp. 56-62, Sep. 2011.

[5] I. I. Kim and E. J. Korevaar, "Availability of free-space optics (FSO) and hybrid FSO/RF systems," in Proc. of SPIE 4530, Opt. Wireless Commun. $I V$, vol. 4530, Nov. 2001, pp. 84-95.

[6] F. Demers, H. Yanikomeroglu, and M. St-Hilaire, "A survey of opportunities for free space optics in next generation cellular networks," in Proc. 9th Annu. Commun. Netw. Services Research Conference (CNSR), May 2011, pp. 210-216.

[7] S. Kazemlou, S. Hranilovic, and S. Kumar, "All-optical multihop freespace optical communication systems," J. Lightw. Technol., vol. 29, no. 18, pp. 2663-2669, Jun. 2011.

[8] M. A. Kashani and M. Uysal, "Outage performance and diversity gain analysis of free-space optical multi-hop parallel relaying," IEEE J. Opt. Commun. Netw., vol. 5, no. 8, pp. 901-909, Aug. 2013.

[9] D. Tsonev, S. Videv, and H. Haas, "Light fidelity (Li-Fi): Towards alloptical networking," in Proc. of SPIE, vol. 9007, 2013, pp. 900 702-1$900702-10$.

[10] H. Burchardt, N. Serafimovski, D. Tsonev, S. Videv, and H. Haas, "VLC: Beyond point-to-point communication," IEEE Commun. Mag., vol. 52, no. 7, pp. 98-105, July 2014.

[11] J. M. Kahn, J. R. Barry, M. D. Audeh, J. B. Carruthers, W. J. Krause, and G. W. Marsh, "Non-directed infrared links for high-capacity wireless LANs," IEEE Personal Commun., vol. 1, no. 2, pp. 12-25, 2nd quarter 1994.

[12] P. Pathak, X. Fen, P. Hu, and P. Mohapatra, "Visible light communication, networking, and sensing: A survey, potential and challenges," IEEE Commun. Surveys Tuts., vol. 17, no. 4, pp. 2047-2077, 4th quarter 2015.
[13] H. Marshoud, V. M. Kapinas, G. K. Karagiannidis, and S. Muhaidat, "Non-orthogonal multiple access for visible light communications," IEEE Photon. Technol. Lett., vol. 28, no. 1, pp. 51-54, Jan 2016.

[14] M. Kashef, M. Abdallah, and K. Qaraqe, "Power allocation for downlink multi-user SC-FDMA visible light communication systems," in 49th Annu. Conf. Infomr. Sci. Syst. (CISS), Mar. 2015, pp. 1-5.

[15] J. Lian and M. Brandt-Pearce, "Distributed power allocation for multiuser MISO indoor visible light communications," in Proc. IEEE Global Commun. Conf. (GLOBECOM), San Diego, CA, Dec. 2015.

[16] H. Ma, L. Lampe, and S. Hranilovic, "Coordinated broadcasting for multiuser indoor visible light communication systems," IEEE Trans. Commun., vol. 63, no. 9, pp. 3313-3324, Sep. 2015.

[17] H. Kazemi and H. Haas, "Downlink cooperation with fractional frequency reuse in DCO-OFDMA optical attocell networks," in Proc. IEEE Int. Conf. Commun. (ICC), Kuala Lumpur, Malaysia, May 2016.

[18] Z. Chen and H. Haas, "Space division multiple access in visible light communications," in IEEE Int. Conf. Commun. (ICC), June 2015, pp. $5115-5119$.

[19] R. C. Kizilirmak, C. R. Rowell, and M. Uysal, "Non-orthogonal multiple access (NOMA) for indoor visible light communications," in Proc. 4th Int. Workshop Opt. Wireless Commun. (IWOW), Sep. 2015, pp. 98-101.

[20] S. S. Bawazir, P. C. Sofotasios, S. Muhaidat, Y. Al-Hammadi, and G. K. Karagiannidis, "Multiple access for visible light communications: Research challenges and future trends," arXiv:1612.05894, 2016.

[21] T. V. Pham and A. T. Pham, "Max-min fairness and sum-rate maximization of MU-VLC local networks," in Proc. IEEE Globecom Workshop Opt. Wireless Commun., San Diego, CA, Dec. 2015.

[22] A. Chaaban, Z. Rezki, and M.-S. Alouini, "On the capacity of the intensity-modulation direct-detection optical broadcast channel," IEEE Trans. Wireless Commun., vol. 15, no. 5, pp. 3114-3130, May 2016.

[23] J. Dang and Z. Zhang, "Comparison of optical OFDM-IDMA and optical OFDMA for uplink visible light communications," in Proc. Int. Conf. Wireless Commun. Signal Process. (WCSP), Oct. 2012, pp. 1-6.

[24] A. Al-Fuqaha, M. Guizani, M. Mohammadi, M. Aledhari, and M. Ayyash, "Internet of things: A survey on enabling technologies, protocols, and applications," IEEE Commun. Surveys Tuts., vol. 17, no. 4, pp. 2347-2376, 4th quarter 2015.

[25] A. Lapidoth, S. M. Moser, and M. Wigger, "On the capacity of freespace optical intensity channels," IEEE Trans. Inf. Theory, vol. 55, no. 10, pp. 4449-4461, Oct. 2009.

[26] A. A. Farid and S. Hranilovic, "Capacity bounds for wireless optical intensity channels with Gaussian noise," IEEE Trans. Inf. Theory, vol. 56, no. 12, pp. 6066-6077, Dec. 2010.

[27] J. M. H. Elmirghani and R. A. Cryan, "Hybrid PPM-CDMA systems utilising OOC for indoor wireless infrared communication," Microwave and Opt. Tech. Lett., vol. 8, no. 1, pp. 44-47, Jan. 1995.

[28] B. M. Ghaffari, M. Matinfar, and J. A. Salehi, "Wireless optical CDMA LAN: Digital design concepts," IEEE Trans. Commun., vol. 56, no. 12, pp. 2145-2155, Dec. 2008.

[29] D. Tse and P. Viswanath, Fundamentals of Wireless Communications. Cambridge University Press, 2005.

[30] R. Ahlswede, "Multi-way communication channels," in Proc. 2nd Int. Symp. Inf. Theory, Tsahkadsor, Armenian S.S.R., Sep. 1971, pp. 23-52. 
[31] H. H. J. Liao, "Multiple Access Channels," Ph.D. dissertation, Department of Electrical Engineering, University of Hawaii, Honolulu, Sep. 1972.

[32] A. Chaaban, J.-M. Morvan, and M.-S. Alouini, "Free-space optical communications: Capacity bounds, approximations, and a new spherepacking perspective," IEEE Trans. Commun., vol. 64, no. 3, pp. 11761191, Mar. 2016

[33] A. A. Farid and S. Hranilovic, "Channel capacity and non-uniform signalling for free-space optical intensity channels," IEEE J. Sel. Areas Commun., vol. 27, no. 9, pp. 1-12, Dec. 2009.

[34] J. R. Barry, J. M. Kahn, W. J. Krause, E. A. Lee, and D. G. Messerschmitt, "Simulation of multipath impulse response for indoor wireless optical channels," IEEE J. Sel. Areas Commun., vol. 11, no. 3, pp. 367379, Apr. 1993.

[35] S. Hranilovic and F. R. Kschischang, "Capacity bounds for power- and band-limited optical intensity channels corrupted by Gaussian noise," IEEE Trans. on Info. Theory, vol. 50, no. 5, pp. 784-795, May 2004.

[36] R. You and J. M. Kahn, "Upper-bounding the capacity of optical IM/DD channels with multiple-subcarrier modulation and fixed bias using trigonometric moment space method," IEEE Trans. Inf. Theory, vol. 48 , no. 2, pp. 514-523, Feb. 2002

[37] S. M. Moser, "Capacity results of an optical intensity channel with inputdependent Gaussian noise," IEEE Trans. Inf. Theory, vol. 58, no. 1, pp. 207-223, Jan. 2012.

[38] A. E. Gamal and Y.-H. Kim, Network Information Theory. Cambridge University Press, 2011.

[39] T. Cover and J. Thomas, Elements of Information Theory (Second Edition). John Wiley and Sons, Inc., 2006.

[40] J. A. Thomas, "Feedback can at most double Gaussian multiple access channel capacity," IEEE Trans. Inf. Theory, vol. 33, no. 5, pp. 711-716, Sep. 1987.

[41] T. H. Chan, S. Hranilovic, and F. R. Kschischang, "Capacity-achieving probability measure for conditionally Gaussian channel with bounded input," IEEE Trans. Inf. Theory, vol. 51, no. 6, pp. 2073-2088, Jun. 2005.

[42] V. V. Prelov and E. C. van der Meulen, "An asymptotic expression for the information and capacity of a multidimensional channel with weak input signals," IEEE Trans. Inf. Theory, vol. 39, no. 5, pp. 1728-1735, Sep. 1993.

[43] A. Chaaban, J.-M. Morvan, and M.-S. Alouini, "Free-space optical communications: Capacity bounds, approximations, and a new sphere-packing perspective," KAUST Technical Report http://hdl.handle.net/10754/552096, Apr. 2015.

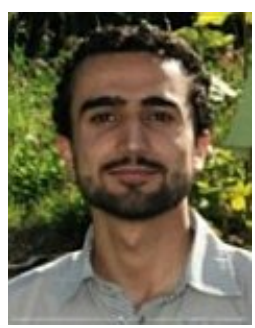

Anas Chaaban (S'09 - M'14) received his Maîtrise ès Sciences degree in electronics from the Lebanese University, Lebanon, in 2006. He received his M.Sc. degree in communications technology and his Dr.Ing. (Ph.D.) degree in Electrical Engineering and Information Technology from the University of Ulm and the Ruhr-University of Bochum, Germany, in 2009 and 2013, respectively. During 2008-2009, he was with the Daimler AG research group on machine vision, Ulm, Germany. He was a Research Assistant with the Emmy- Noether Research Group on Wireless Networks at the University of Ulm, Germany, during 2009-2011, which relocated to Ruhr-Universität Bochum, Germany, in 2011. He was a postdoctoral researcher at the Ruhr-Universität Bochum, Germany, in 20132014, and joined King Abdullah University of Science and Technology as a postdoctoral researcher in 2015. His research interests are in the areas of information theory and wireless communications.
Omer S. Al-Ebraheemy Omer Mahmoud S. Al-Ebraheemy received B.Sc. degree (with First Class Honors) in Electrical Engineering from University of Khartoum (UofK), Khartoum, Sudan in 2004, and the M.Sc. degree (with Highest Distinction) in Electrical Engineering from Blekinge Institute of Technology (BTH), Blekinge, Sweden in 2012. He has over 5 years of experience working with wireless cellular network operators and system vendors in $2 \mathrm{G} \& 3 \mathrm{G}$ Radio Network Planning and Optimization. He is currently a lecturer-B and working towards the Ph.D. degree in electrical engineering at King Fahd University of Petroleum and Minerals (KFUPM), Dhahran, Saudi Arabia. His research interests include signal processing, information theory, and wireless communications. He was the recipient of the Best Academic Performance Award in Faculty of Engineering, University of Khartoum, ZAIN Aim for Excellence Award for exceptional performance in developing the DDS Feature in their cellular network in 2009, and the Ph.D. Scholarship from KFUPM in 2013.

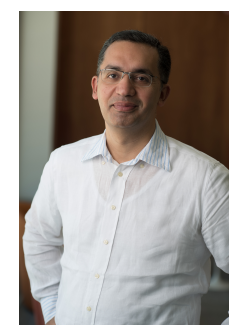

Tareq Y. Al-Naffouri (M'10) Tareq Al-Naffouri received the B.S. degrees in mathematics and electrical engineering (with first honors) from King Fahd University of Petroleum and Minerals, Dhahran, Saudi Arabia, the M.S. degree in electrical engineering from the Georgia Institute of Technology, Atlanta, in 1998, and the Ph.D. degree in electrical engineering from Stanford University, Stanford, CA, in 2004. He was a visiting scholar at California Institute of Technology, Pasadena, CA, from January to August 2005 and during summer 2006. He was a Fulbright scholar at the University of Southern California from February to September 2008. He has held internship positions at NEC Research Labs, Tokyo, Japan, in 1998, Adaptive Systems Lab, University of California at Los Angeles in 1999, National Semiconductor, Santa Clara, CA, in 2001 and 2002, and Beceem Communications Santa Clara, CA, in 2004. He is currently an Associate Professor at the Electrical Engineering Department, King Abdullah University of Science and Technology (KAUST). His research interests lie in the areas of sparse, adaptive, and statistical signal processing and their applications and in network information theory. He has over 170 publications in journal and conference proceedings, 9 standard contributions, 11 issued patents, and 5 pending. Dr. Al-Naffouri is the recipient of the IEEE Education Society Chapter Achievement Award in 2008 and Al-Marai Award for innovative research in communication in 2009. Dr. Al-Naffouri has also been serving as an Associate Editor of Transactions on Signal Processing since August 2013.

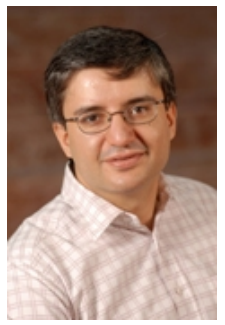

Mohamed-Slim Alouini (S'94, M'98, SM'03, F09) was born in Tunis, Tunisia. He received the Ph.D. degree in Electrical Engineering from the California Institute of Technology (Caltech), Pasadena, CA, USA, in 1998. He served as a faculty member in the University of Minnesota, Minneapolis, MN, USA, then in the Texas A\&M University at Qatar, Education City, Doha, Qatar before joining King Abdullah University of Science and Technology (KAUST), Thuwal, Makkah Province, Saudi Arabia as a Professor of Electrical Engineering in 2009. His current research interests include the modeling, design, and performance analysis of wireless communication systems. 